International Journal for Numerical and Analytical Methods in Geomechanics

Volume 41, Issue 1, 1 January 2017, Pages 30-53, DOI: 10.1002/nag.2544

\title{
A basal slip model for Lagrangian finite element simulations of 3D landslides
}

\author{
Massimiliano Cremonesi ${ }^{1 *}$, Francesco Ferri ${ }^{1}$ and Umberto Perego ${ }^{1}$ \\ ${ }^{1}$ Department of Civil and Environmental Engineering, Politecnico di Milano, Italy
}

\begin{abstract}
SUMMARY
A Lagrangian numerical approach for the simulation of rapid landslide runouts is presented and discussed. The simulation approach is based on the so called Particle Finite Element Method (PFEM). The moving soil mass is assumed to obey a rigid-viscoplastic, non-dilatant Drucker-Prager constitutive law, which is cast in the form of a regularized, pressure sensitive Bingham model. Unlike in classical formulations of computational fluid mechanics, where no-slip boundary conditions are assumed, basal slip boundary conditions are introduced to account for the specific nature of the landslide-basal surface interface. The basal slip conditions are formulated in the form of modified Navier boundary conditions, with a pressure sensitive threshold. A special mixed Eulerian-Lagrangian formulation is used for the elements on the basal interface to accommodate the new slip conditions into the PFEM framework. To avoid inconsistencies in the presence of complex shapes of the basal surface, the no-flux condition through the basal surface is relaxed using a penalty approach. The proposed model is validated by simulating both laboratory tests and a real large scale problem, and the critical role of the basal slip is elucidated.
\end{abstract}

KEY WORDS: slip boundary conditions, landslide simulation, PFEM, Lagrangian approach

\section{INTRODUCTION}

The triggering of a landslide can be induced by natural causes (erosion, heavy rainfall, earthquake) or by human actions (excavation, construction, mining). The propagation velocity is affected by the slope conformation, the material and the failure mechanism. Landslides characterized by high velocities and long runout distances behave in a fluid-like manner. Modeling such fluidized material is important for the creation of maps of hazardous areas, to estimate the entity of the hazard and finally to design appropriate protective measures. The prediction of runout distances and velocities requires a complete mathematical modeling of the phenomenon.

In the literature, approaches for the simulation of a propagating landslide usually belong to one of two main groups: approaches based on discrete models and approaches based on continuum models. The Discrete Element Method (DEM) is a typical example of discrete models. The landslide is described by a set of particles of different shape interacting with each other through contact forces. The material constitutive behavior plays a role only in the contact between particles making the definition of the forces the crucial part of the method. Examples of the use of DEM for the simulation of landslide can be found e.g. in [1, 2, 3]. In [4] a Molecular Dynamics approach, an alternative discrete scheme, has been proposed for modeling the triggering and propagation of landslides caused by rainfall.

Continuum models treat the landslide material as a continuum medium. Starting from the balance equations (mass, momentum and energy conservation), models with different levels of complexity

\footnotetext{
${ }^{*}$ Correspondence to: Department of Civil and Environmental Engineering, Politecnico di Milano, piazza Leonardo da Vinci 32, 20133 Milano, Italy. E-mail: massimiliano.cremonesi@ polimi.it
} 
can be considered. These equations can be formulated for all the involved phases (solid particles, fluid and gas) [5, 6], or for one single homogenized phase representing the material overall behavior.

A common simplification in continuum models consists of integrating the equations of motion along the vertical axis [7]. The 2D depth-integrated model is simple and can provide accurate results in terms of velocity of propagation, time to reach a particular place and depth of the flow. These models have been widely used to describe flow-like landslides $[8,9,10,11]$. However, in the case of high velocities and accelerations in the depth direction or rough or irregular basal surfaces, depth integrated models can hardly be applicable. Details about depth-averaged models and a complete bibliography can be found in the recent overview [12].

From the numerical point of view, starting from the equations of motion of the continuum problem, different methods have been proposed. Smoothed Particle Hydrodynamics (SPH), originally conceived for astrophysical problems, has been successfully applied to model catastrophic landslides [11]. In particular, the group of Pastor has studied different aspects of the use of SPH for landslide simulations (see for example $[13,12]$ and the references therein).

Methods based on structured and unstructured grids have also been proposed. In [14, 15], applications of the Finite Volume method have been proposed, while in [16, 17], Finite Difference approaches have been used. Also the Finite Element Method has been extensively used. In [18] a Lagrangian finite element method has been formulated to reproduce the dynamic runout caused by a landslide. In [19] an Arbitrary Lagrangian-Eulerian approach is used to model entrainment/deposition phenomena in rock and debris avalanches. [10] proposes a finite element discretization of the depth-averaged equations using a two step Taylor-Galerkin algorithm. [20] presents a numerical model, based on Material Point Method (MPM), for studying the dynamic evolution of landslides. In this case the combination of the Lagrangian and the Eulerian descriptions allows for the modeling of complex material behavior in the presence of large displacements.

A constitutive model is required by all continuum approaches. A common approach consists of modeling the complex landslide material as a fluid governed by simple internal and basal rheological properties, described by a very limited number of parameters (see e.g. [21]). Of course, the objectives achievable with these simple models are limited to some macroscopic information, such as duration and runout distance, flow depth and velocity in the region of the impact against existing civil structures and infrastructures, extension of the final deposit. This is however the most relevant information for risk assessment. In this case, rather then on laboratory measurements, parameters are calibrated on a sufficiently large number of prototype real cases, through back analysis, defining suitable parameter ranges, applicable to events having similar macroscopic characteristics (see e.g. [22] for a discussion on parameter calibration for this type of problems). Constitutive behaviors can be based on elasto-plastic and/or rheological models. The Bingham model is the simplest model to describe the transition between solid and fluid phases (see e.g. [23] for a comparison with other rheological models). When the shear stress is below a certain threshold, the material behaves like a rigid body, whereas the material flows like a viscous fluid when the limit is exceeded. In frictional models, the threshold stress varies with the pressure field and the friction angle [10]. A cohesion can also be added to this model [24]. More complex models can also include effects like erosion [25].

This work is devoted to the presentation of a numerical approach for the three-dimensional simulation of landslide runout starting from an unstable slope. A landslide is here intended as a gravity-driven free surface flow (granular flow, mud flow, debris flow, etc.). The numerical analysis of this type of problems requires the ability to track free surfaces and interfaces, in the case that more than one material (e.g. a landslide impinging in a water reservoir [26]) is involved, to account for complex constitutive behaviors and possibly for multi-physics phenomena. The need of simulating evolving free surfaces and interfaces makes Eulerian approaches less convenient to use in this context. A Lagrangian approach with continuous remeshing, to account for the extremely large deformations and consequent mesh distortion, based on the so-called Particle Finite Element Method (PFEM) [27] is here adopted. The PFEM is a finite element method originally developed for the simulation of fluid dynamics, including free surface flows and breaking waves [28], and fluidstructure interaction problems $[29,30,31]$. The method has been applied and validated on a large 
number of different problems, including simulations of landslides [32] and of landslide generated water waves $[26,33]$.

No-slip boundary conditions are usually assumed in the solution of Navier-Stokes equations for fluid dynamics problems. However, wall slip is known to occur in many industrial processes, such as in polymer extrusion [34], and the definition of appropriate slip boundary conditions is still the object of discussion [35]. In the case of granular flows, the slip velocity between the flowing mass and the basal surface has been observed to increase with the average grain diameter in [36], where the velocity profiles in granular landslides have been experimentally studied. As a consequence of the frictional relative slip, a new basal dissipation mechanism need be characterized in addition to the viscous dissipation inside the material bulk. This is usually done introducing a basal friction coefficient, which however is not easy to identify for real landslides. A recent and comprehensive study [37] has shown that the effective friction coefficient is not a constant property of the interface, but decreases with increasing the slip velocity and total mass of the moving landslide. This frictional weakening can possibly be produced by micro-mechanical processes such as flash-heating, whereby frictional heating is produced at the micro-contact scale. For high slip velocities, heat dissipation is prevented and thermal weakening effects like melting or other phase transformations and phenomena are activated [38, 39, 40, 41].

The consideration of slip boundary conditions together with Navier-Stokes equations is not standard. The classical no-slip boundary condition in fluid dynamics is macroscopically acceptable in most cases and is based on physical and mathematical considerations at the microscale [42], but it is not physically acceptable in several types of liquid flow at solid surfaces [43] and, as already mentioned, for increasing diameter granular flows [36]. The simplest, classical alternative to the no-slip boundary conditions are the so-called Navier boundary conditions, that establish a linear dependence, through a material friction-like parameter, of the basal tangential stress on the slip velocity. In practice, one can imagine a variety of different conditions, passing from maximum tangential stress and no-slip, to zero tangential stress and free slip in tangential direction.

A convenient geometrical parameter to describe this variety of conditions is the slip length [44]. The meaning of the slip length is shown in Figure 1, where a Couette flow between two rigid walls at a distance $h$ is shown. The upper wall is moving with assigned horizontal velocity $U$, while the lower wall is fixed. The case of perfect no-slip condition is shown in Figure 1a. In Figure 1b a certain amount of slip is present, with a non-zero fluid tangential velocity $u_{s l i p}$ at the lower plate interface $(y=0)$. The slip length $h_{\text {slip }}$ is defined as the distance from the lower plate to the point at zero velocity obtained extrapolating the linear velocity profile. Figure 1c shows the limit case of free slip, where an infinite slip length is obtained. Negative slip lengths can also occur in some cases [44] when the lower fluid layers are locked to the plate and the no-slip condition extends to some layers inside the fluid channel. The slip length can be considered to be a property of the fluid-wall interface.

a)

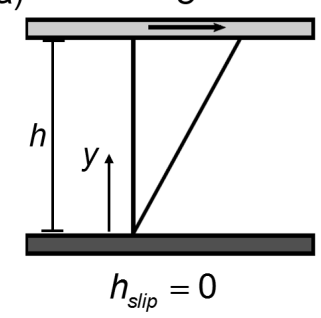

b)

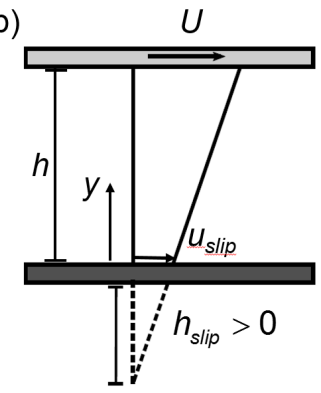

c)

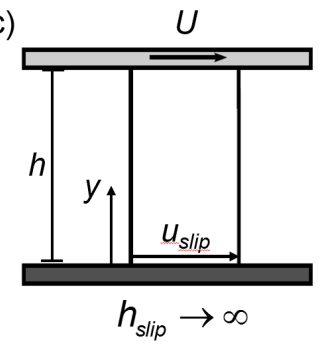

Figure 1. Definition of slip length $h_{\text {slip }}$ for a Couette flow. Top plate (light gray) moves with prescribed horizontal velocity, while bottom plate (dark gray) is fixed. Velocity profiles are shown for different situations: a) no-slip $\left(h_{\text {slip }}=0\right)$; b) slip $\left(h_{\text {slip }}>0\right)$; c) free slip $\left(h_{\text {slip }} \rightarrow \infty\right)$.

In practical cases, the slip often occurs only when a critical value of the tangential stress is reached, in analogy with the classical Bingham model. The Navier boundary condition can then be 
easily modified by adding a constant threshold to the linear function of the slip velocity. However, from the mathematical point of view, this implies a significant complication since the weak form of the balance equations has to be formulated as a variational inequality [45]. To avoid this difficulty, a regularization similar to the one usually adopted for Bingham flows $[46,26]$ is here adopted. The regularized slip condition can be thought of as a non-linear Navier boundary condition, where the non-linearity is due, e.g., to a stress dependent slip length (see e.g. [47] for the formulation of a Newtonian flow with non-linear Navier boundary condition).

Defining by $\mathbf{n}$ the unit normal to the basal interface and by $\mathbf{u}$ the velocity at a point, the imposition of slip boundary conditions requires that also the no-flux condition $\mathbf{u} \cdot \mathbf{n}=\mathbf{0}$ is enforced. These conditions are known to lead to the so called Babuska's paradox [48], with non-convergent approximations in the presence of curved boundaries, where polygonal finite element discretizations are used. Other difficulties connected with this type of boundary conditions, especially in combination with the incompressibility constraint, are the non-unique definition of the normal vector at boundary nodes with piecewise linear discretization (see [49] for a technique for the computation of a unique normal, based on mass conservation; see also [50] for a discussion) and the particular way in which the evolving boundary conditions are treated in the PFEM with continuous remeshing. To alleviate these problems, in this work the no-flux condition is enforced through a penalization technique following [51], together with a reduced integration of the penalty integral term.

In summary, in the present paper a Lagrangian finite element tool, based on the PFEM, for runout simulation of three-dimensional landslides is discussed. The flowing material is assumed to be incompressible and to obey a Drucker-Prager type of rigid-viscoplastic constitutive law. This is shown to be equivalent to adopt a frictional Bingham-like behavior. The balance equations are formulated in terms of velocities as in Navier-Stokes equations. Non-standard slip boundary conditions are introduced to account for the basal slip of the moving landslide. A simple model for the description of the interaction between the flowing soil mass and the basal surface, based on a Coulomb friction model, where the tangential stress at the interface is set in relation with the slip velocity, is proposed. This requires the definition of an effective basal friction coefficient and of a slip length to characterize the interface. An Arbitrary Lagrangian-Eulerian formulation [52] on the basal interface, together with a penalization of the no-flux condition through the basal surface are used to accommodate the slip condition into the PFEM framework. Both the rigid-viscoplastic bulk law and the basal friction law are regularized to achieve a variational statement of the balance equations without variational inequalities. The tool is applied to the simulation of a number of both laboratory and real scale landslides.

\section{LARGE DEFORMATION KINEMATICS AND MATERIAL MODELING}

Landslide runouts involve extremely large deformations of the moving soil mass. For this reason, in the literature they have been modeled mainly by considering the soil as a viscous fluid. This assumption implies that the initial, statically equilibrated state cannot be included in the model and the transition from stable slope configuration to an unstable moving landslide cannot be described.

In this work, the moving mass is modeled as a rigid-viscoplastic solid undergoing large deformations. This implies that also in this case only the landslide motion can be described. The assumed rigid-viscoplastic behavior is obtained by simply neglecting the elastic part of the behavior of an elastic-viscoplastic material model. In contrast, the modeling of the soil evolution from its initially stable equilibrium configuration to the final unstable state of running landslide will require consideration of the complete elasto-viscoplastic material behavior.

\subsection{Kinematics}

Consider a soil mass with initial configuration $\mathcal{B}_{0}$ occupying the volume $\Omega_{0}$ with boundary $\partial \Omega_{0}$. The body undergoes a motion described by a deformation mapping $\chi(\mathbf{X}, t)$, where $\mathbf{X}$ denotes the position of a material particle in the reference configuration, while the corresponding position in 
the current configuration $\mathcal{B}_{t}$ at time $t$ occupying the volume $\Omega_{t}$ with boundary $\partial \Omega_{t}$, is defined by $\mathbf{x}=\chi(\mathbf{X}, t)$.

Let $\mathbf{F}=\nabla_{X}$ be the deformation gradient. Its polar decomposition in terms of the left stretch tensor $\mathbf{V}$ and of the rotation tensor $\mathbf{R}$ is given by:

$$
\mathbf{F}=\mathbf{V R}
$$

Let $\mathbf{B}=\mathbf{V} \mathbf{V}^{T}$ be the left Cauchy-Green (or Finger) tensor and $J=\operatorname{det} \mathbf{F}$. Defining the isotropic $\operatorname{logarithmic}$ strain tensor $\varepsilon=\ln \mathbf{V}=\frac{1}{3} \ln \mathbf{B}$ and the deviatoric part $\mathbf{V}_{D}$ of $\mathbf{V}$ as $\mathbf{V}_{D}=J^{-1 / 2} \mathbf{V}$, one obtains the additive decomposition of the logarithmic strain ( $\mathrm{I}$ being the identity tensor):

$$
\boldsymbol{\varepsilon}=\varepsilon_{D}+\mathbf{I} \varepsilon_{V} \quad \text { with } \quad \varepsilon_{V}=\operatorname{tr} \varepsilon=\ln J, \varepsilon_{D}=\ln \mathbf{V}_{D}
$$

The usual multiplicative decomposition of the deformation gradient $\mathbf{F}$ into its elastic $\mathbf{F}^{e}$ and plastic $\mathbf{F}^{p}$ parts is assumed [53]:

$$
\mathbf{F}=\mathbf{F}^{e} \mathbf{F}^{p} \quad \mathbf{F}^{e}=\mathbf{V}^{e} \mathbf{R}^{e}, \mathbf{F}^{p}=\mathbf{V}^{p} \mathbf{R}^{p}
$$

with obvious meaning of symbols. According to this decomposition, the spatial velocity gradient $\mathbf{l}=\nabla_{x} \mathbf{u}=\dot{\mathbf{F}} \mathbf{F}^{-1}, \mathbf{u}$ denoting the velocity field, is expressed as:

$$
\mathbf{l}=\mathbf{l}^{e}+\mathbf{F}^{e} \mathbf{L}^{p} \mathbf{F}^{e-1}=\mathbf{l}^{e}+\mathbf{l}^{p}
$$

where

$$
\mathbf{l}^{e}=\dot{\mathbf{F}}^{e} \mathbf{F}^{e-1}, \quad \mathbf{L}^{p}=\dot{\mathbf{F}}^{p} \mathbf{F}^{p-1}
$$

define the spatial elastic and plastic velocity gradients, respectively. Note that while $\mathbf{l}^{p}$ is defined in the current configuration, $\mathbf{L}^{p}$ is defined in the intermediate configuration. The plastic velocity gradient in the current configuration is given by $\mathbf{l}^{p}=\mathbf{F}^{e} \mathbf{L}^{p} \mathbf{F}^{e-1}$.

The elastic and plastic velocity gradients $\mathbf{l}^{e}$ and $\mathbf{l}^{p}$ can be split in the sum of their symmetric and antisymmetric components:

$$
\begin{aligned}
& \mathbf{l}^{e}=\frac{1}{2}\left(\mathbf{l}^{e}+\mathbf{l}^{e T}\right)+\frac{1}{2}\left(\mathbf{l}^{e}-\mathbf{l}^{e T}\right)=\mathbf{d}^{e}+\mathbf{w}^{e} \\
& \mathbf{l}^{p}=\frac{1}{2}\left(\mathbf{l}^{p}+\mathbf{l}^{p T}\right)+\frac{1}{2}\left(\mathbf{l}^{p}-\mathbf{l}^{p T}\right)=\mathbf{d}^{p}+\mathbf{w}^{p}
\end{aligned}
$$

where $\mathbf{d}^{e}, \mathbf{d}^{p}$ define the elastic and plastic rate of deformation tensors, and $\mathbf{w}^{e}, \mathbf{w}^{p}$ the corresponding spin tensors. As it is customary in plastically isotropic solids, $\mathbf{w}^{p}=0$ is assumed, and therefore:

$$
\mathbf{l}=\mathbf{d}^{e}+\mathbf{d}^{p}+\mathbf{w}^{e}=\nabla_{x} \mathbf{u}
$$

where $\mathbf{u}$ is the velocity of a material particle and $\nabla_{x}$ is the gradient spatial operator computed with respect to the current configuration.

\subsection{Material model}

Let $\sigma$ be the Cauchy stress tensor and $\tau=J \sigma$ the Kirchhoff stress tensor. The deviatoric components of the Cauchy and Kirchhoff stresses are given by

$$
\boldsymbol{\sigma}_{D}=\boldsymbol{\sigma}+\mathbf{I} p, \quad \boldsymbol{\tau}_{D}=J \boldsymbol{\sigma}_{D}
$$

where $p=-\operatorname{tr} \boldsymbol{\sigma}$ is the hydrostatic stress component, taken positive in compression.

Elastic strains in soils are usually small, so that a Hencky type linear relation is assumed between Kirchhoff stresses and logarithmic strains

$$
\boldsymbol{\tau}_{D}=\rho_{0} \frac{\partial \Psi}{\partial \boldsymbol{\varepsilon}^{e}}=2 G \boldsymbol{\varepsilon}_{D}^{e}+K \varepsilon_{V}^{e} \mathbf{I}
$$


where $\varepsilon^{e}=\ln \mathbf{V}^{e}, \rho_{0}$ is the density in the reference configuration, $G$ is the shear modulus, $K$ the bulk modulus and

$$
\Psi\left(\varepsilon^{e}\right)=\frac{1}{2} \varepsilon^{e}: \mathbf{E}: \varepsilon^{e}
$$

is the free energy potential per unit mass, $\mathbf{E}$ being the constant isotropic fourth order elasticity tensor.

The elastic domain is assumed to be bounded by a Drucker-Prager like condition with zero cohesion

$$
q-\alpha J p \tan \varphi \leq 0
$$

where $q=\sqrt{\frac{3}{2} \tau: \tau}$ is the Kirchhoff effective stress, $\alpha$ is a material constant and $\varphi$ is the material internal friction angle, which is assumed here to be constant. A non-associative, non-dilatant viscoplastic flow rule is assumed, whereby the purely deviatoric plastic strain rate is expressed as the gradient of a viscoplastic potential $g(\boldsymbol{\tau})=q(\boldsymbol{\tau})$

$$
\mathbf{d}^{p}=\frac{f}{\mu} \frac{\partial g}{\partial \boldsymbol{\tau}}=\frac{3}{2 \mu} \frac{f}{q} \boldsymbol{\tau}_{D}
$$

where $\mu$ is the material viscosity and $f$ is a measure of the overstress

$$
f=<q-\alpha J p \tan \varphi>
$$

with $<\square>=\square$ for $\square>0,<\square>=0$ for $\square \leq 0$. With these definitions the plastic work density is obtained as

$$
\boldsymbol{\tau}: \mathbf{d}^{p}=\boldsymbol{\tau}_{D}: \mathbf{d}^{p}=\frac{3}{2 \mu} \frac{f}{q} \boldsymbol{\tau}_{D}: \boldsymbol{\tau}_{D}=q \dot{\gamma}, \quad \dot{\gamma}=\frac{f}{\mu}
$$

where $\dot{\gamma}$ is the effective plastic strain rate, conjugate to $q$ in the plastic work.

After the conditions for the slope unstable motion have been met, the landslide runout starts, with negligible elastic strains if compared to the extremely large viscoplastic deformations. In this case, it is customary to assume $\mathbf{d}^{e}=0$ in (8). Since plastic strain rates are purely deviatoric, the granular flow behaves as an incompressible non-Newtonian fluid with $J=1$ and $\boldsymbol{\tau}_{D}=\boldsymbol{\sigma}_{D}$. Under these conditions, from (13) one can express the deviatoric Kirchhoff stress $\boldsymbol{\tau}_{D}$ in terms of the plastic strain rate $\mathbf{d}^{p}$

$$
\boldsymbol{\tau}_{D}=\frac{2}{3} \frac{q}{\dot{\gamma}} \mathbf{d}^{p}, \quad f>0
$$

But further noting that for $f>0$ and $J=1$, from the expression (15) 2 of $\dot{\gamma}$ and making use of (14), $q$ can be expressed as

$$
q=\mu \dot{\gamma}+\alpha p \tan \varphi, \quad f>0
$$

one finally obtains

$$
\boldsymbol{\sigma}_{D}=\boldsymbol{\tau}_{D}=\frac{2}{3}\left(\mu+\frac{\alpha p \tan \varphi}{\dot{\gamma}}\right) \mathbf{d}^{p}, \quad f>0
$$

The expression in (18) defines a Bingham-like frictional rigid viscoplastic behavior and is substantially identical to the one considered e.g. in [10]. This can be regularized [46, 26] defining an effective viscosity $\tilde{\mu}$ accounting for the frictional contributions.

$$
\tilde{\mu}=\frac{2}{3}\left[\mu+\frac{\alpha p \tan \varphi}{\dot{\gamma}}\left(1-e^{-N \dot{\gamma}}\right)\right]
$$

where $N$ is a regularization parameter, usually taken equal to $10^{3}$, and setting

$$
\boldsymbol{\sigma}_{D}=\boldsymbol{\tau}_{D}=\tilde{\mu} \mathbf{d}
$$

for every arbitrary $f$, where it has been set $\mathbf{d}^{e}=\mathbf{0}, \mathbf{d}=\mathbf{d}^{p}$ and $J=1$. With the additional assumption of $\alpha=1$, this the the expression that will be used in what follows. 


\section{GOVERNING EQUATIONS}

\subsection{Balance equations and boundary conditions}

The motion of the rigid-viscoplastic material discussed in the previous section is described using Navier-Stokes equations for incompressible fluids in an Arbitrary Lagrangian-Eulerian framework [52]:

$$
\begin{array}{rr}
\rho\left(\frac{\partial \mathbf{u}}{\partial t}+\left(\mathbf{c} \cdot \nabla_{x}\right) \mathbf{u}\right)=\nabla_{x} \cdot\left(\boldsymbol{\sigma}_{D}-p \mathbf{I}\right)+\rho \mathbf{b} & \text { in } \Omega_{t} \times(0, T) \\
\nabla_{x} \cdot \mathbf{u}=0 & \text { in } \Omega_{t} \times(0, T)
\end{array}
$$

where $\nabla_{x}$ is the gradient spatial operator computed with respect to the current configuration and the convective velocity $\mathbf{c}$ is defined as:

$$
\mathbf{c}=\mathbf{u}-\mathbf{r}
$$

$\mathbf{u}$ being the velocity of material particles and $\mathbf{r}$ the mesh velocity. In general, an additional equation governing the evolution of the mesh $\mathbf{r}$ is necessary. The standard Eulerian description of the equation of motion can be recovered imposing $\mathbf{r}=0$ (i.e. $\mathbf{c}=\mathbf{u}$ ). Conversely, the Lagrangian description is obtained enforcing $\mathbf{r}=\mathbf{u}$ (i.e. $\mathbf{c}=0$ ). As a consequence, in the Eulerian approach the mesh is fixed while in the Lagrangian one the mesh moves with the velocity of the fluid particles.

Equations (21) need be supplemented with proper initial and boundary conditions. The boundary $\partial \Omega_{t}$ is partitioned into three non-overlapping subsets $\partial \Omega_{t}=\Gamma_{t}^{D} \cup \Gamma_{t}^{N} \cup \Gamma_{t}^{S}$. On $\Gamma_{t}^{D}$ and $\Gamma_{t}^{N}$ standard Dirichlet and Neumann boundary conditions are imposed while on $\Gamma_{t}^{S}$ slip boundary conditions are considered. On $\Gamma_{t}^{D}$ Dirichlet boundary conditions are imposed on velocities and on $\Gamma_{t}^{N}$ Neumann boundary conditions are imposed on surface tractions:

$$
\begin{aligned}
\mathbf{u}(\mathbf{x}, t) & =\overline{\mathbf{u}}(\mathbf{x}, t) & & \text { on } \Gamma_{t}^{D} \\
\boldsymbol{\sigma}(\mathbf{x}, t) \cdot \mathbf{n} & =\mathbf{h}(\mathbf{x}, t) & & \text { on } \Gamma_{t}^{N}
\end{aligned}
$$

where $\overline{\mathbf{u}}(\mathbf{x}, t)$ and $\mathbf{h}(\mathbf{x}, t)$ are assigned functions and $\mathbf{n}$ is the outward normal to the boundary.

Let $\mathbf{u}_{\text {slip }}$ be the relative velocity between the moving soil and the basal surface $\Gamma_{t}^{S}$ and $\mathbf{t}$ the tangential traction acting on the landslide material along the same surface (Figure 2):

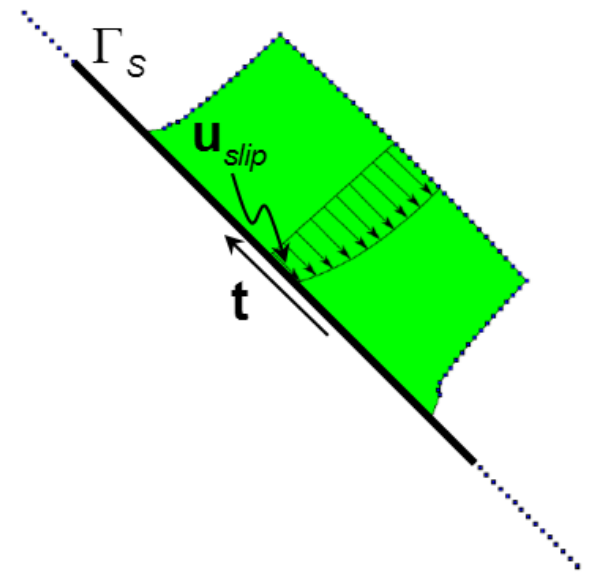

Figure 2. Slip velocity profile and basal tangential traction.

$$
\mathbf{u}_{\text {slip }}=(\mathbf{I}-\mathbf{n} \otimes \mathbf{n}) \mathbf{u}, \quad \mathbf{t}=(\mathbf{I}-\mathbf{n} \otimes \mathbf{n})\left[\left(\boldsymbol{\sigma}_{D}-p \mathbf{I}\right) \mathbf{n}\right]
$$


Navier type boundary conditions with a pressure dependent threshold are assumed along $\Gamma_{t}^{S}$ :

$$
\mathbf{u}_{\text {slip }}=-\beta \frac{\mathbf{t}}{\|\mathbf{t}\|}\left\langle\|\mathbf{t}\|-p \tan \varphi_{\text {basal }}\right\rangle
$$

where $\beta=h_{\text {slip }} / \mu$ is a parameter, having the dimension of a length over a viscosity, characterizing the basal interface, $h_{\text {slip }}$ is the slip length for an ideal Couette flow with no threshold $\left(\varphi_{\text {basal }}=0\right)$ and $\varphi_{\text {basal }}$ is the friction angle of the basal interface. In the case of equation (25), with a threshold on the tangential traction, the interface is characterized by two parameters, $h_{s l i p}($ or $\beta)$ and $\varphi_{\text {basal }}$, since $\mu$ is taken equal to the bulk viscosity. Note that in this case $h_{\text {slip }}$ cannot be interpreted as the zero velocity position of the velocity profile, since this position is not constant in a Couette flow with slip threshold. Condition (25) states that the slip is resisted by a tangential force proportional to the slip velocity. For $\beta=0$, the no-slip boundary condition is recovered, while $\beta \rightarrow \infty$ represents the stress free boundary condition. According to (25), slippage occurs only when the pressure dependent threshold value $p \tan \varphi_{\text {basal }}$ is exceeded by the tangential traction $\mathbf{t}$.

From (25) one has

$$
\beta\left\langle\|\mathbf{t}\|-p \tan \varphi_{\text {basal }}\right\rangle=\left\|\mathbf{u}_{\text {slip }}\right\|
$$

and solving for $\|\mathbf{t}\|$

$$
\|\mathbf{t}\|=\frac{1}{\beta}\left\|\mathbf{u}_{\text {slip }}\right\|+p \tan \varphi_{\text {basal }} \quad \text { for } \quad\|\mathbf{t}\|-p \tan \varphi_{\text {basal }}>0
$$

Replacing the expression (26) in (25) and solving for $\mathbf{t}$ one has

$$
\mathbf{t}=-\frac{\|\mathbf{t}\|}{\left\|\mathbf{u}_{\text {slip }}\right\|} \mathbf{u}_{\text {slip }} \quad \text { for } \quad\|\mathbf{t}\|-p \tan \varphi_{\text {basal }}>0
$$

Finally, replacing the expression (27) of $\|\mathbf{t}\|$ one obtains (see e.g. [45])

$$
\mathbf{t}=-\left(\frac{1}{\beta}+\frac{p \tan \varphi_{\text {basal }}}{\left\|\mathbf{u}_{\text {slip }}\right\|}\right) \mathbf{u}_{\text {slip }} \text { for }\|\mathbf{t}\|-p \tan \varphi_{\text {basal }}>0
$$

The expression in (29) is formally similar to the expression in (18) and it can be regularized using the same technique:

$$
\mathbf{t}=-\frac{1}{\tilde{\beta}} \mathbf{u}_{\text {slip }}, \quad \frac{1}{\tilde{\beta}}=\frac{1}{\beta}+\frac{p \tan \varphi_{\text {basal }}}{\left\|\mathbf{u}_{\text {slip }}\right\|}\left(1-e^{-N\left\|\mathbf{u}_{\text {slip }}\right\|}\right)
$$

The simple expression in $(30)_{1}$, which is equivalent to adopting a non-linear Navier type boundary condition with stress dependent slip length, has been used to model the relation between the slip velocity and the tangential traction acting along the basal interface.

With these definitions, the complete set of boundary conditions consists of (23) on $\Gamma_{t}^{D}$ and $\Gamma_{t}^{N}$, supplemented by the conditions on $\Gamma_{t}^{S}$, that include the no-flux condition through the basal interface:

$$
\left.\begin{array}{l}
\mathbf{t}=-\frac{1}{\tilde{\beta}} \mathbf{u}_{s l i p} \\
\mathbf{u} \cdot \mathbf{n}=0
\end{array}\right\} \quad \text { on } \quad \Gamma_{t}^{S}
$$

\subsection{Variational form of the balance equations}

Without loss of generality, in the following $\mathbf{h}(\mathbf{x}, t)=0$ and $\overline{\mathbf{u}}(\mathbf{x}, t)=0$ will be assumed on $\Gamma_{t}^{N}$ and $\Gamma_{t}^{D}$, respectively. The weak form of the balance equations (21), with the boundary conditions (23) and (31) is obtained following a standard Galerkin approach:

$$
\begin{aligned}
\int_{\Omega_{t}} \rho\left[\frac{\partial \mathbf{u}}{\partial t}+\left(\mathbf{c} \cdot \nabla_{x}\right) \mathbf{u}\right] \cdot \mathbf{v} d \Omega_{t}-\int_{\Omega_{t}}\left(\nabla_{x} \cdot \boldsymbol{\sigma}+\rho \mathbf{b}\right) \cdot \mathbf{v} d \Omega_{t} & +\int_{\Gamma^{N}}(\boldsymbol{\sigma} \mathbf{n}) \cdot \mathbf{v} d \Gamma+ \\
& +\int_{\Gamma^{S}}\left(\mathbf{t}+\frac{1}{\tilde{\beta}} \mathbf{u}_{s l i p}\right) \cdot \mathbf{v} d \Gamma=0 \\
\int_{\Omega_{t}}\left(\nabla_{x} \cdot \mathbf{u}\right) \phi d \Omega_{t}=0 &
\end{aligned}
$$


where $\mathbf{v}$ and $\phi$ are respectively vector and scalar test functions, with $\mathbf{v}=\mathbf{0}$ on $\Gamma^{D}, \mathbf{v} \cdot \mathbf{n}=0$ on $\Gamma^{S}, \phi=0$ on $\Gamma^{N}$. Integrating by parts the second integral in $(32)_{1}$, the usual variational form is recovered

$$
\begin{aligned}
\int_{\Omega_{t}} \rho\left[\frac{\partial \mathbf{u}}{\partial t}+\left(\mathbf{c} \cdot \nabla_{x}\right) \mathbf{u}\right] \cdot \mathbf{v} d \Omega_{t}+\int_{\Omega_{t}} \boldsymbol{\sigma}_{D}: \nabla_{x} \mathbf{v} d \Omega_{t} & -\int_{\Omega_{t}} p\left(\nabla_{x} \cdot \mathbf{v}\right) d \Omega_{t}-\int_{\Omega_{t}} \rho \mathbf{b} \cdot \mathbf{v} d \Omega_{t}+ \\
& \int_{\Gamma^{S}}\left(-\mathbf{\sigma n}+\mathbf{t}+\frac{1}{\tilde{\beta}} \mathbf{u}_{s l i p}\right) \cdot \mathbf{v} d \Gamma=0 \\
\int_{\Omega_{t}}\left(\nabla_{x} \cdot \mathbf{u}\right) \phi d \Omega_{t}=0 &
\end{aligned}
$$

Noting that

$$
\int_{\Gamma^{S}}(-\boldsymbol{\sigma n}+\mathbf{t}) \cdot \mathbf{v} d \Gamma=-\int_{\Gamma^{S}} \mathbf{v} \cdot(\mathbf{n} \otimes \mathbf{n})(\boldsymbol{\sigma n}) d \Gamma=-\int_{\Gamma^{S}}(\mathbf{v} \cdot \mathbf{n}) \mathbf{n} \cdot \boldsymbol{\sigma} \mathbf{n} d \Gamma=0
$$

the variational form of the balance equations is finally written as

$$
\begin{gathered}
\int_{\Omega_{t}} \rho\left[\frac{\partial \mathbf{u}}{\partial t}+\left(\mathbf{c} \cdot \nabla_{x}\right) \mathbf{u}\right] \cdot \mathbf{v} d \Omega_{t}+\int_{\Omega_{t}} \boldsymbol{\sigma}_{D}: \nabla_{x} \mathbf{v} d \Omega_{t}-\int_{\Omega_{t}} p\left(\nabla_{x} \cdot \mathbf{v}\right) d \Omega_{t}-\int_{\Omega_{t}} \rho \mathbf{b} \cdot \mathbf{v} d \Omega_{t}+ \\
\int_{\Omega_{t}}\left(\nabla_{x} \cdot \mathbf{u}\right) \phi d \Omega_{t}=0
\end{gathered}
$$

As noted by several authors (see e.g. [48, 49, 50]), the enforcement of the no-flux condition $(31)_{2}$ in the presence of curved boundaries may lead to numerical inconsistencies. For this reason, the no-flux condition is here replaced by a penalized form, in the line of what proposed in [51], together with a reduced integration of the penalty integral term. Let us set

$$
\mathbf{n} \cdot \boldsymbol{\sigma} \mathbf{n}=-\frac{1}{\epsilon} \mathbf{u} \cdot \mathbf{n}
$$

on $\Gamma^{S}, \epsilon$ being a small number. With this definition, the last integral in $(32)_{1}$ becomes

$$
\int_{\Gamma^{S}}\left[\left(\mathbf{t}+\frac{1}{\tilde{\beta}} \mathbf{u}_{\text {slip }}\right)+\left(\mathbf{n} \cdot \boldsymbol{\sigma} \mathbf{n}+\frac{1}{\epsilon} \mathbf{u} \cdot \mathbf{n}\right) \mathbf{n}\right] \cdot \mathbf{v} d \Gamma
$$

where now $\mathbf{v}$ is not required anymore to satisfy $\mathbf{v} \cdot \mathbf{n}=\mathbf{0}$ on $\Gamma^{S}$. After integration by parts and taking into account that $\mathbf{t}+(\mathbf{n} \cdot \boldsymbol{\sigma n}) \mathbf{n}=\boldsymbol{\sigma n}$, the last integral in (33) $)_{1}$ transforms into

$$
\int_{\Gamma^{S}}\left[-\boldsymbol{\sigma n}+\left(\mathbf{t}+\frac{1}{\tilde{\beta}} \mathbf{u}_{\text {slip }}\right)+\left(\mathbf{n} \cdot \boldsymbol{\sigma n}+\frac{1}{\epsilon} \mathbf{u} \cdot \mathbf{n}\right) \mathbf{n}\right] \cdot \mathbf{v} d \Gamma=\int_{\Gamma^{S}}\left[\frac{1}{\tilde{\beta}} \mathbf{u}_{\text {slip }}+\frac{1}{\epsilon}(\mathbf{u} \cdot \mathbf{n}) \mathbf{n}\right] \cdot \mathbf{v} d \Gamma
$$

\subsection{Space discretization}

The weak form (33), with the modified conditions (38) on the slip boundary $\Gamma^{S}$, is discretized for implementation in the Particle Finite Element Method (PFEM) [27]. This is a Lagrangian method, based on triangular (2D) or tetrahedral (3D) elements, with continuous remeshing consisting of a fast Delaunay re-triangulation with prescribed node positions. When remeshing is performed, data have to be transmitted from the old mesh to the new one. Since elements connectivities are modified, while nodes remain the same to save computing time and to avoid accuracy loss, pressure and velocity are both modeled in terms of nodal quantities using linear interpolation functions, leading 
to an unstable method that requires a stabilization [54]. Details about the stabilization procedure adopted in this work can be found in $[31,55]$.

A key point in the PFEM is the algorithm for boundary identification and for contact detection. This is based on the so called alpha-shape method (see e.g. [27, 31] for details), which removes unnecessary elements from the mesh using a criterion based on mesh distortion. In a Lagrangian framework, the external boundary and the current volume are defined by the position of the material particles. Every time that the mesh is regenerated with the Delaunay triangulation, the particles belonging to the boundary may change and the new boundary nodes (and therefore the particles) have to be identified. The identification process is divided into two phases : i) identification of the real shape of the particle distribution; ii) identification of the nodes that belong to the boundary. The second phase is almost trivial, but the first one is critical. The Delaunay triangulation generates the convex hull of the set of particles, which, however, does not conform to the external boundary where this is not convex. The key idea of the alpha-shape method is to remove the unnecessary elements from the mesh using a criterion based on the mesh distortion. For each element $e$ of the mesh, the minimum distance $d^{e}$ between two nodes in the element and the radius $R^{e}$ of its circumcircle are defined. If $h^{e}$ is computed as the mean value of all the $d^{e}$, the shape factor $\alpha^{e}=R^{e} / h^{e}$ is an index of the element distortion. All the elements that do not satisfy the condition $\alpha^{e} \leq \bar{\alpha}$ are removed from the mesh, where $\bar{\alpha} \geq 1$ is assumed. Increasing the value of $\bar{\alpha}$, fewer elements are removed from the original mesh and, for $\bar{\alpha} \rightarrow \infty$, the original Delaunay triangulation is recovered. Once the unnecessary elements are removed, the particles belonging to the actual boundary can be identified.

In the case of landslides runout, the computational domain consists of the initial volume occupied by the soil mass plus the whole surface of the slope on which the landslide can possibly slide during its runout. In the spirit of the method, nodes are placed on the whole sliding surface, so that finite elements can be generated automatically by the remeshing procedure whenever the soil mass is approaching that part of the boundary. These nodes are not active, and therefore are not identified as material particles (i.e. they do not possess any degree of freedom), until they become the vertex of a finite element of sufficiently regular shape (i.e. that is not removed by the alpha-shape method).

Slip boundary conditions are difficult to enforce in this fully Lagrangian framework, in which nodes on the basal surface have to move according to the soil velocity, but at the same time have to define the position of the boundary. If slip nodes are moved following their velocity, the definition of the boundary is lost and the boundary tracking algorithm cannot work anymore. To overcome this difficulty, all nodes in the mesh are treated as Lagrangian (i.e. $\mathbf{c}=\mathbf{0}$ in (21)) except those on $\Gamma^{S}$ (where slip conditions are imposed), which are treated as Eulerian (i.e. $\mathbf{c}=\mathbf{u}$ ) and therefore remain fixed in their initial position. This special treatment gives rise to a mixed Lagrangian-Eulerian formulation, whereby some nodes are Lagrangian and some others (more specifically those on $\Gamma^{S}$ ) are Eulerian. In particular, elements having a node on the boundary $\Gamma^{S}$ will have degrees of freedom of mixed nature (Figure 3).

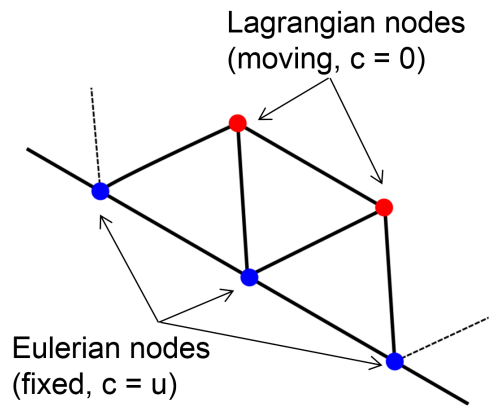

Figure 3. Schematic illustration of the distinction between Lagrangian and Eulerian nodes. 
Proceeding now with a standard finite element discretization with linear interpolation functions for both pressure and velocity, the following semidiscretized form is obtained:

$$
\begin{array}{r}
\mathbf{M U}+\left(\mathbf{K}+\mathbf{K}_{\text {slip }}+\mathbf{K}_{c}\right) \mathbf{U}+\mathbf{D}^{T} \mathbf{P}=\mathbf{B} \\
\mathbf{D U}=\mathbf{0}
\end{array}
$$

where (see [31] for a detailed Lagrangian definition of $\mathbf{K}$ and $\mathbf{D}$ ) $\mathbf{U}$ and $\mathbf{P}$ contain the nodal values of velocity and pressure respectively, $\mathbf{M}$ is the mass matrix, $\mathbf{K}$ is the matrix of viscoplastic coefficients, having the same structure of the deviatoric part of the stiffness matrix, $\mathbf{D}$ is the discretization of the divergence operator, $\mathbf{B}$ is the vector of body forces and boundary tractions, $\mathbf{K}_{c}$ is the discretization of the convective term on the slip boundary and $\mathbf{K}_{\text {slip }}$ is the discretization of the integral in (38). Their definition is given below.

Let $n_{\text {dim }}$ define the problem dimension $\left(n_{\text {dim }}=2\right.$ in $2 \mathrm{D}, n_{\text {dim }}=3$ in $\left.3 \mathrm{D}\right)$ and let $n_{\text {node }}$ be the number of nodes per element $\left(n_{\text {node }}=3\right.$ for triangular elements, $n_{\text {node }}=4$ for tetrahedra). Defining with $\Omega_{t}^{S}$ the subdomain of $\Omega_{t}$ containing elements with at least one node on $\Gamma^{S}$ and setting $\mathbf{c}^{e}(\mathbf{x})=\mathbf{N}^{e}(\mathbf{x}) \mathbf{C}^{e}, \mathbf{C}^{e}$ being nodal values of the velocity $\mathbf{c}(\mathbf{x})$ on element $\Omega^{e}$, the components of matrix $\mathbf{K}_{c}^{e}$ are obtained as:

$$
\left[\mathbf{K}_{c}^{e}\right]_{a_{h} b_{k}}=\int_{\boldsymbol{\Omega}^{e} \in \Omega^{S}} N_{a, j}^{e} C_{d j}^{e} N_{d}^{e} N_{b}^{e} \delta_{h k} d \Omega^{e}
$$

where $a, b, d=1, \ldots n_{\text {node }}, i, j, h, k=1 \ldots n_{\text {dim }}, \delta_{h k}$ is the Kronecker delta, $N_{a}$ is the shape function of node $a, N_{a, j}=\partial N_{a} / \partial \mathbf{x}_{j}$, and summation on repeated indexes $d$ and $j$ is assumed. In (40), $\left[\mathbf{K}_{c}^{e}\right]_{a_{h} b_{k}}$ denotes the $h k$ component of the submatrix (of dimensions $n_{\operatorname{dim}} \times n_{\operatorname{dim}}$ ) $\left[\mathbf{K}_{c}^{e}\right]_{a b}$, $C_{d j}^{e}$ is the $j$-th component of $\mathbf{c}$ at node $d$, with $C_{d j}^{e}=0$ if $d$ is an interior node (Lagrangian node in Figure 3) and $C_{d j}^{e}=U_{d j}^{e}$ if $d$ is a node on $\Gamma^{S}$ (Eulerian node in Figure 3).

$\mathbf{K}_{\text {slip }}$ for element $e$ is defined as:

$$
\mathbf{K}_{\text {slip }}^{e}=\int_{\Gamma_{e}^{S}} \mathbf{Q}^{e T} \mathbf{N}^{e T}\left[\begin{array}{ccc}
\frac{1}{\epsilon} & 0 & 0 \\
0 & \frac{1}{\tilde{\beta}} & 0 \\
0 & 0 & \frac{1}{\tilde{\beta}}
\end{array}\right] \mathbf{N}^{e} \mathbf{Q}^{e} d \Gamma
$$

where $\mathbf{N}^{e}$ is the matrix gathering the shape functions of element $e$ and $\mathbf{Q}^{e}=\operatorname{diag}\left[\mathbf{Q}_{a}^{e}\right], a=$ $1, \ldots n_{\text {node }}$, is a block diagonal matrix. For $a$ denoting a node on $\Gamma^{S}, \mathbf{Q}_{a}^{e}$ is the orthogonal matrix transforming the velocity components $u_{x}, u_{y}, u_{z}$ with respect to the global reference system into the components $u_{n}, u_{t_{1}}, u_{t_{2}}$, locally normal and tangent to the basal surface at each element node $a$ on $\Gamma^{S}$ :

$$
\left[\begin{array}{l}
u_{n} \\
u_{t_{1}} \\
u_{t_{2}}
\end{array}\right]_{a}=\mathbf{Q}_{a}\left[\begin{array}{l}
u_{x} \\
u_{y} \\
u_{z}
\end{array}\right]_{a}
$$

In contrast, for $a$ denoting an interior node, one has $\mathbf{Q}_{a}^{e}=\mathbf{0}$.

All matrices $\mathbf{K}_{c}^{e}$ and $\mathbf{K}_{\text {slip }}^{e}$ not pertaining to elements with nodes on $\Gamma^{S}$ are zero.

\subsection{Time integration and solution scheme}

Considering the time interval $(0, T)$ to be divided into a fixed number of time steps $\Delta T$ and assuming that the state of the system is known at $t=t^{n}$ in terms of nodal positions $\mathbf{x}^{n}=$ $\mathbf{x}\left(t^{n}\right)$, velocities $\mathbf{U}^{n}=\mathbf{U}\left(t^{n}\right)$ and pressures $\mathbf{P}^{n}=\mathbf{P}\left(t^{n}\right)$, the state at time $t=t^{n+1}$ is determined enforcing equations (39) at $t=t^{n+1}$ using a backward Euler integration scheme, with $\dot{\mathbf{U}}^{n+1}=$ $\left(\mathbf{U}^{n+1}-\mathbf{U}^{n}\right) / \Delta t$. The final fully discretized nonlinear problem writes:

$$
\begin{array}{r}
\left(\frac{\mathbf{M}}{\Delta t}+\mathbf{K}+\mathbf{K}_{\text {slip }}+\mathbf{K}_{c}\right) \mathbf{U}^{n+1}+\mathbf{D}^{T} \mathbf{P}^{n+1}= \\
\mathbf{B}+\frac{\mathbf{M}}{\Delta t} \mathbf{U}^{n} \\
\mathbf{D U}^{n+1}=\mathbf{0}
\end{array}
$$


It is important to recall that all matrix and vector operators depend non-linearly on the current configuration and are therefore non-linear functions of the unknown vector $\mathbf{U}^{n+1}$. Iterations are performed within each time step until convergence within the prescribed tolerance is achieved. At each new iteration, all matrices are updated on the basis of the configuration reached at the end of the previous iteration. No tangent operators are used.

In this work, the system of equations in (43) has been solved with a monolithic approach, using the PSPG stabilization proposed by Tezduyar et al. in [55].

\section{NUMERICAL EXAMPLES}

\subsection{Couette flow}

The analytical solutions for Couette and Poiseuille flows with linear and non-linear Navier slip laws have been presented in [56]. To validate the implementation of the slip boundary conditions, a simple test is considered, consisting in a Couette flow of water $\left(\rho=10^{-3} \mathrm{~kg} / \mathrm{m}^{3}, \mu=10^{-3} \mathrm{~Pa} \mathrm{~s}\right.$, $\varphi=\varphi_{\text {basal }}=0$ ) between two parallel plates at distance $h$, where the top plate moves with a fixed velocity $U$, while at the bottom the Navier slip condition is imposed (Figure 1). The analytical solution consists of a linear velocity profile along the channel height:

$$
u(y)=\frac{U(y-h)}{h+h_{\text {slip }}}+U
$$

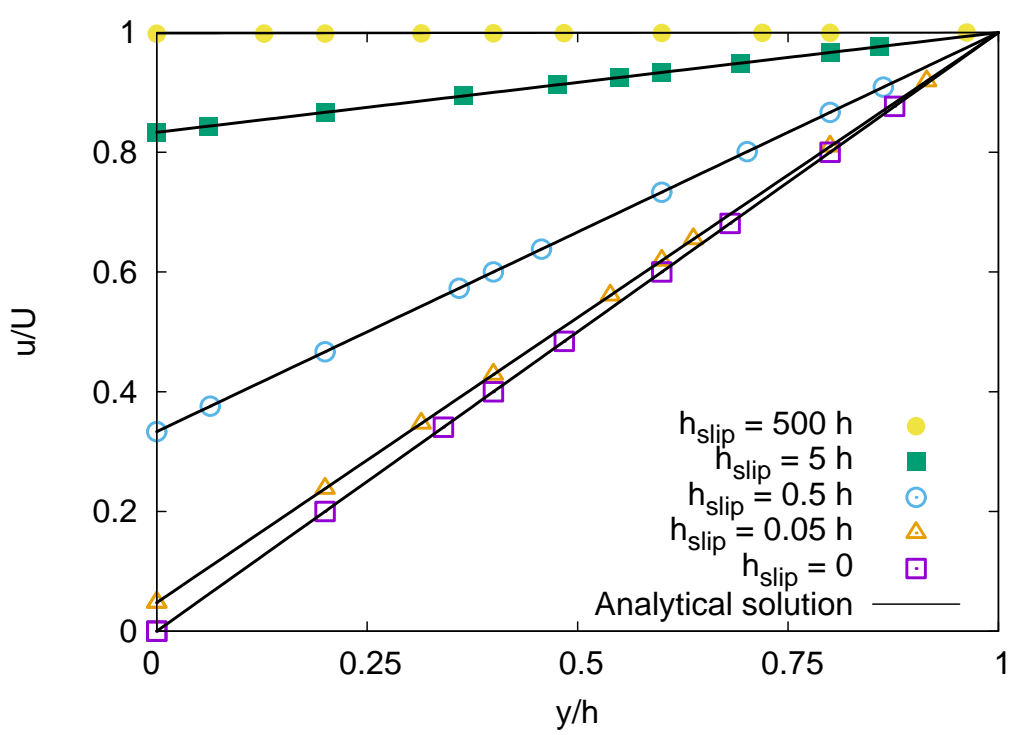

Figure 4. Couette flow. Comparisons between analytical and numerical solution.

Figure 4 shows a comparison between the analytical and the numerical solutions, the latter obtained using the formulation discussed in section 3, varying the slip parameter $h_{\text {slip }}$. A perfect match with the expected solutions is obtained.

\subsection{Chute of a granular mass along inclined planes}

Two experimental tests performed by Manzella at the Ecole Polytechnique Fédéral de Lausanne are considered [57]. The tests consist of the free chute of a granular material along inclined planes. Two different geometries, with one or two inclined planes, are considered (see Figure 5). The inclination of the plane in the first test (Figure 5(a)) is $45^{\circ}$, the same as in the first plane of the second test (Figure 5(b)). 


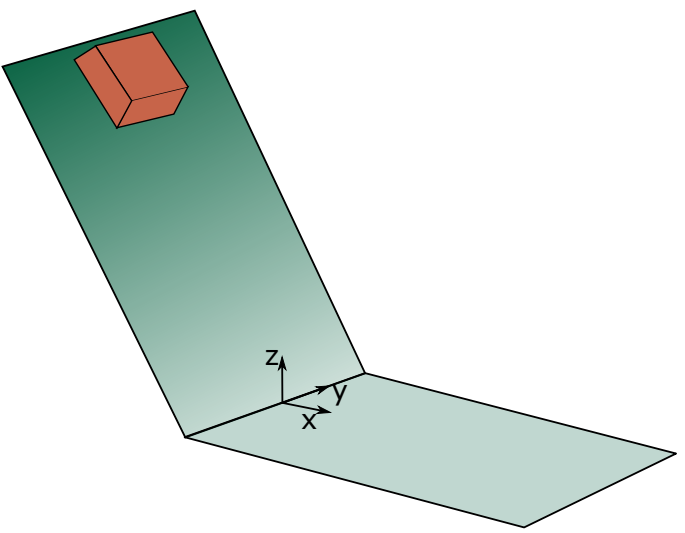

(a)

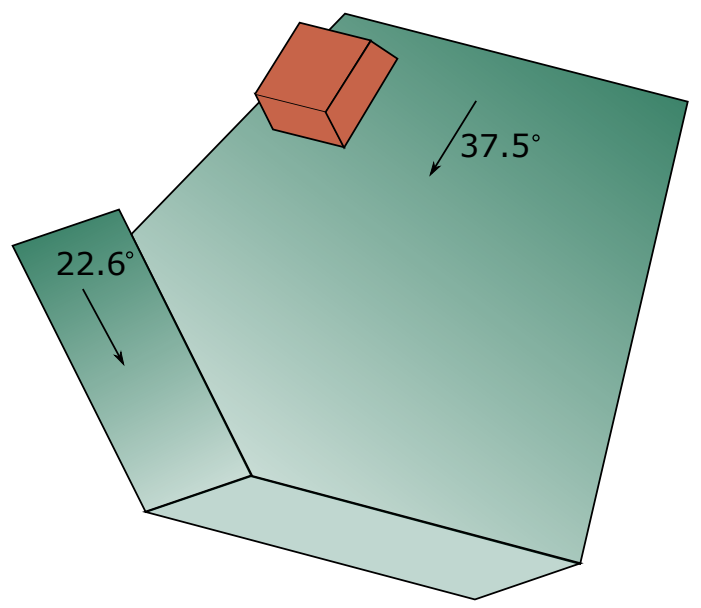

(b)

Figure 5. Chute of a granular mass along inclined planes. Tests setup and granular mass initial positions.

The granular material is a Hostun sand. Material parameters, as provided in [57], are: density $\rho=1280 \mathrm{Kg} / \mathrm{m}^{3}$, friction angle $\varphi=34^{\circ}$ and basal friction angle $\varphi_{\text {basal }}=32^{\circ}$. The total volume of sand is $0.02 \mathrm{~m}^{3}$ for the first test and $0.03 \mathrm{~m}^{3}$ for the second. The second test has also been simulated in $[13,12]$ using the same constitutive parameters. The constitutive model discussed in the previous sections needs two additional parameters: the initial viscosity $\mu$ and the amount of $\operatorname{slip} \beta$ (or $h_{\text {slip }}$ alternatively). These parameters are identified on the basis of the first test then used in the second one. An initial mesh of 240902 tetrahedra with 95422 nodes has been used for all the simulation of these tests, with an average element size of $h^{e}=0.01 \mathrm{~m}$ (see Figure 6). A zero initial velocity has been prescribed for all nodes in the mesh.

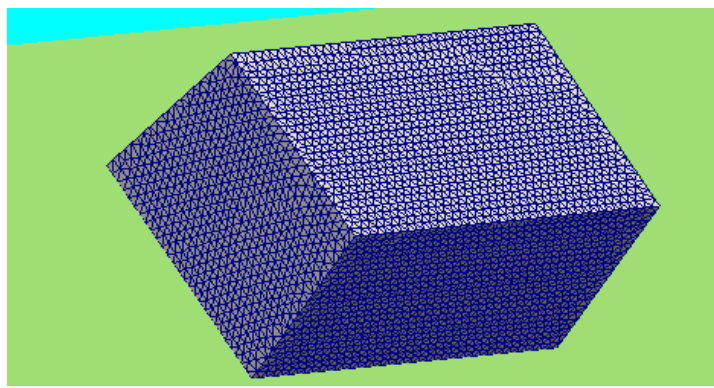

Figure 6. Chute of a granular mass along inclined planes. One inclined plane. Initial mesh used in the simulations.

Experimental and numerical final deposits for the first test (one inclined plane) are compared in Figure 7 and Figure 8. Figure 8(a) shows a view of the final deposit in the $x-y$ plane (see Figure 5 for the used reference system), while its vertical section in the $x-z$ plane is shown in Figure 8(b). The two graphs show the effect of the amount of slip at the interface $\left(h_{\text {slip }}\right)$ on the final results. The final deposit obtained at $t=2.3 \mathrm{~s}$ with no slip condition $\left(h_{\text {slip }}=0\right)$ differs significantly from the experimental data. A good agreement has been obtained setting $\mu=1 \mathrm{~Pa} \mathrm{~s}$ and $h_{\text {slip }}=0.04 \mathrm{~m}$, with a ratio $h_{\text {slip }} / h^{e}=4$.

This first example has been also used for a preliminary study on the possible correlation between the mesh size and slip parameter. Figure 9 shows the final deposit obtained with two different meshes with an average element size $h^{e}=0.01 \mathrm{~m}$ and $h^{e}=0.02 \mathrm{~m}$, respectively, and the same $h_{\text {slip }}=0.04$ $\mathrm{m}$. It can be observed that the results of the finest mesh, on which the slip parameter has been calibrated, are closer to the experimental data. On the contrary, in Figure 10 the slip parameter $h_{\text {slip }}$ 

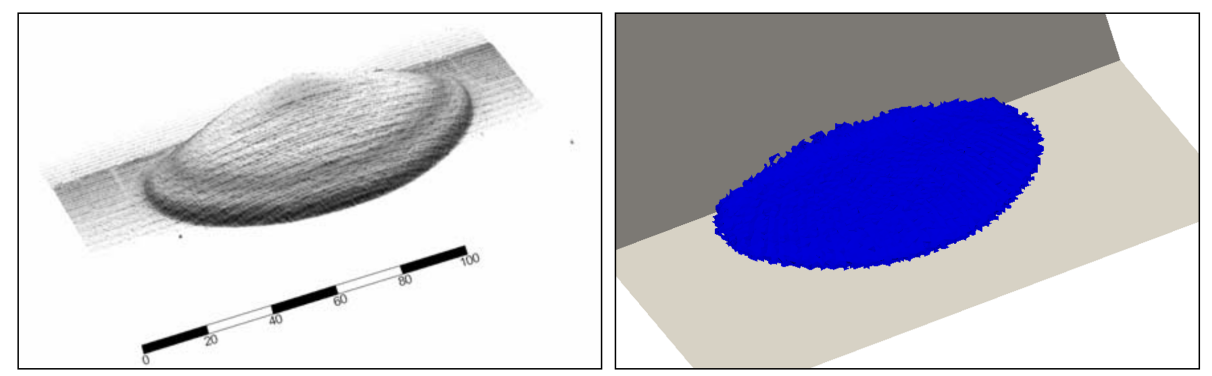

Figure 7. Chute of a granular mass along inclined planes. One inclined plane. Final deposit at $t=2.3 \mathrm{~s}$ : experimental (left) and numerical (right), with $\mu=1 \mathrm{~Pa} \mathrm{~s}, h_{\text {slip }}=0.04 \mathrm{~m}$.

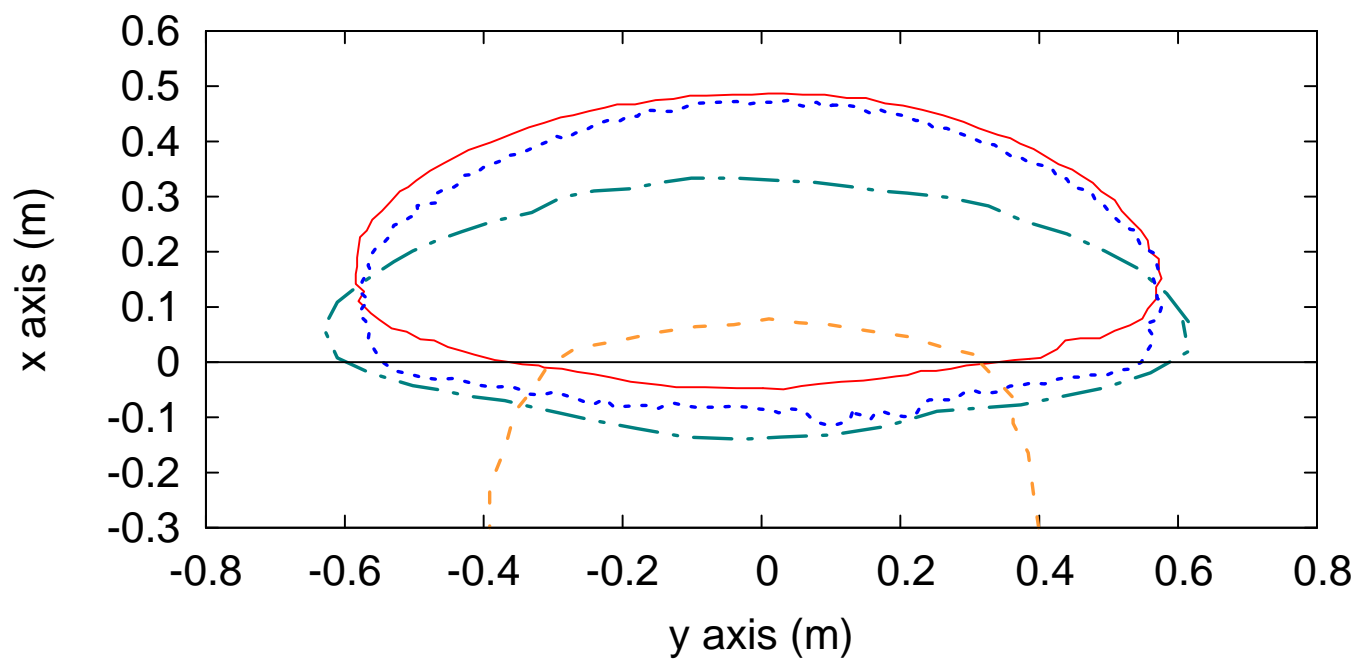

(a) top view

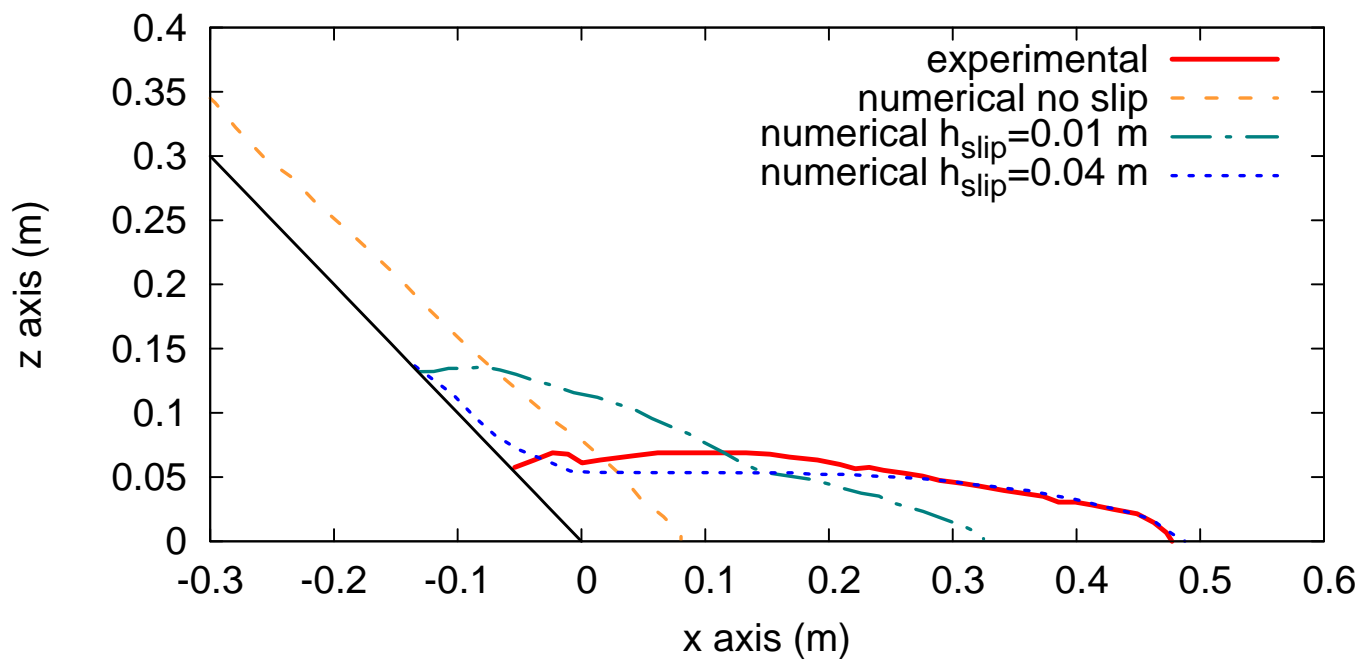

(b) section

Figure 8. Chute of a granular mass along inclined planes. One inclined plane. Comparison of the final deposit with $\mu=1 \mathrm{~Pa} \mathrm{~s}$, at $t=2.3 \mathrm{~s}$. 
has been calibrated separately for the two meshes. It can be argued that for fine meshes, the $h_{\text {slip }}$ parameter does not depend on the mesh size, but only on the interface properties. In contrast, for coarse meshes the slip parameter should account for the mesh coarseness, to better describe the motion in proximity of the basal interface.

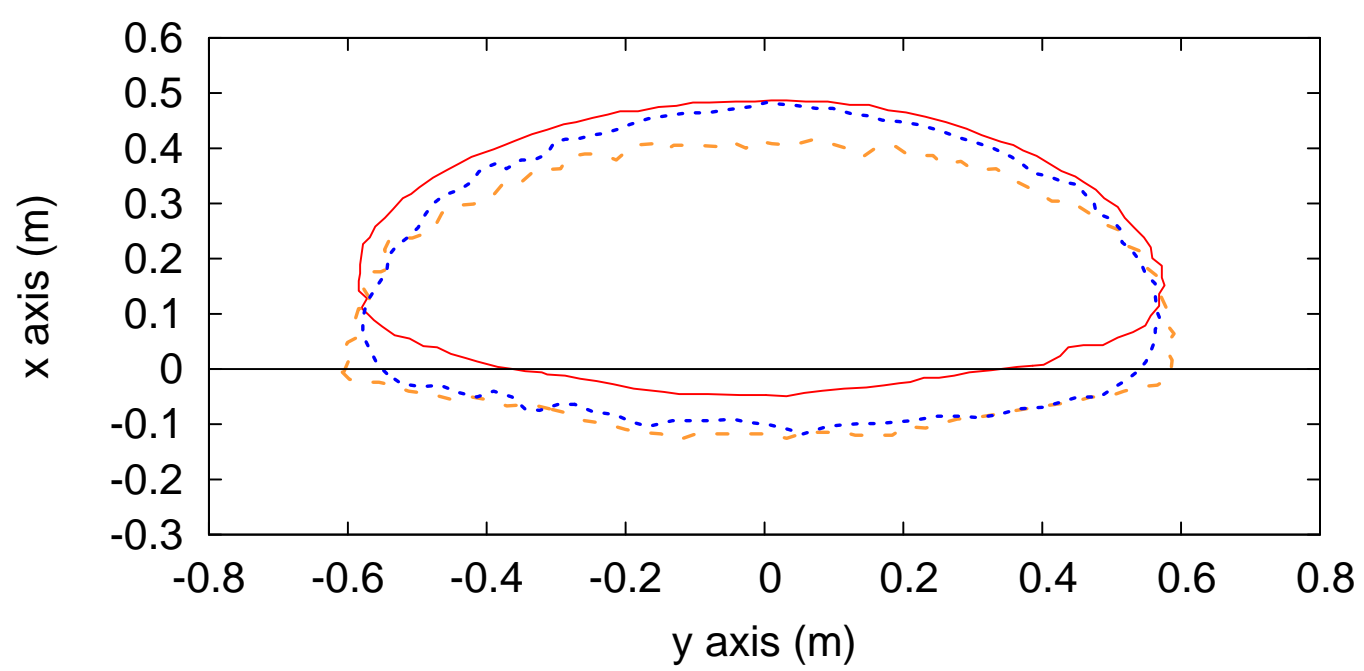

(a) top view

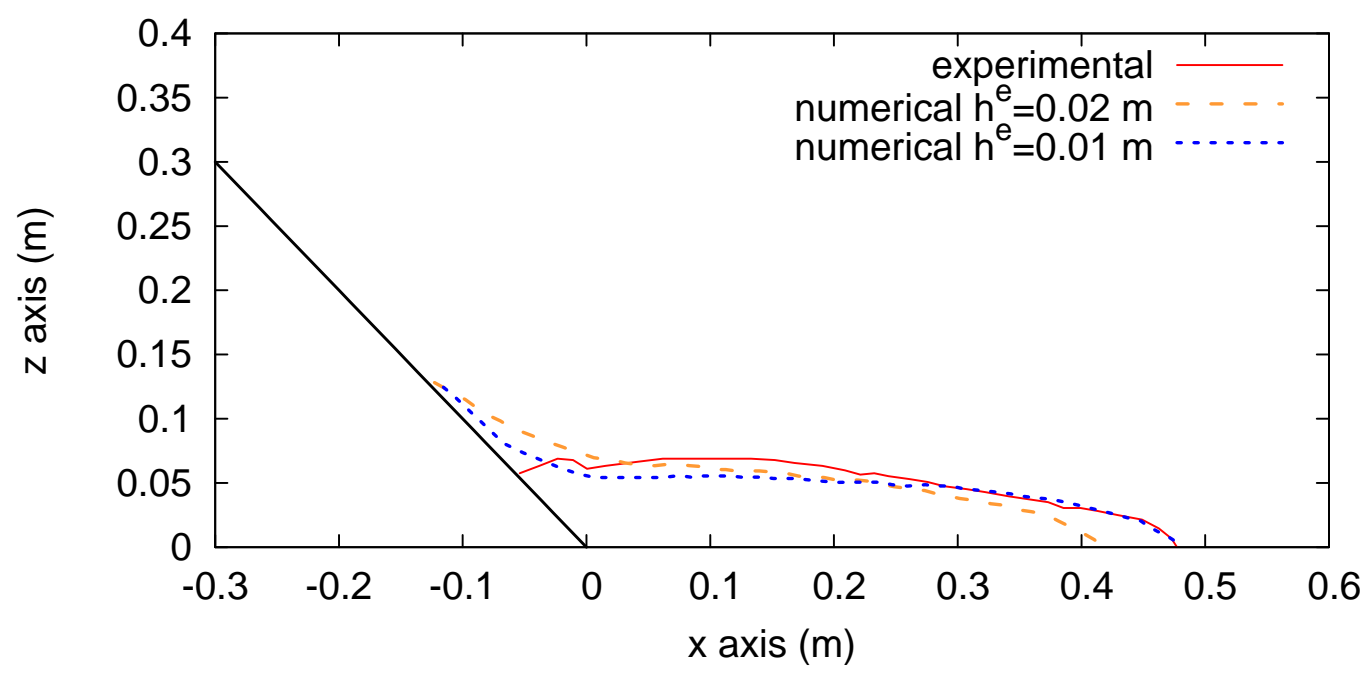

(b) section

Figure 9. Chute of a granular mass along inclined planes. One inclined plane. Comparison of the final deposit varying the average mesh size with $h_{\text {slip }}=0.04 \mathrm{~m}$.

As previously discussed, the no-flux condition has been imposed in a penalized form depending on a penalty parameter $\epsilon$. At the intersection between the inclined and the horizontal planes there is a sharp corner where the no-flux condition need be enforced. The effect of the penalization parameter on the numerical results can be appreciated in Figure 11, where velocity vectors at two instants are plotted in the corner region. Dark gray vectors refer to $\epsilon=10^{5}$ while light gray ones to $\epsilon=10^{7}$. In the first snapshot, taken at the time of the impact, the instantaneous velocity has a non-zero outward normal component to the boundary. The penalization tends to to reduce this component, as it can be appreciated in the second snapshot, taken at a subsequent instant, where the normal component is 


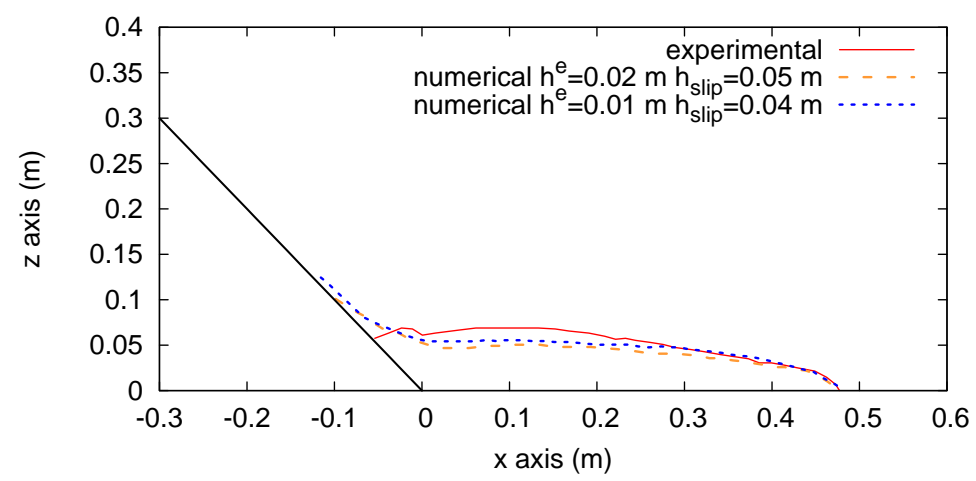

Figure 10. Chute of a granular mass along inclined planes. One inclined plane. Comparison of the final deposit varying the average mesh size and $h_{\text {slip }}$.

much smaller. As expected, larger values of $\epsilon$ more strictly enforce the respect of the non-penetration condition. In all the following examples, $\epsilon=10^{7}$ will be used.

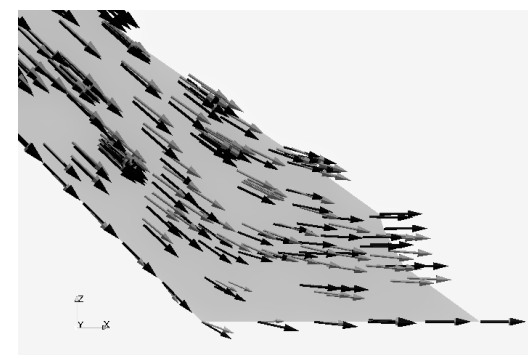

(a)

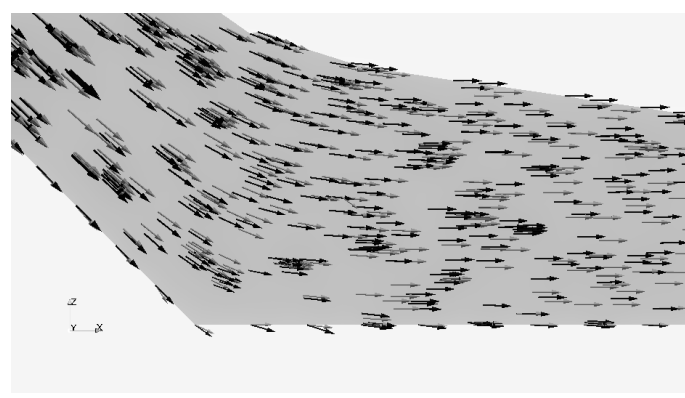

(b)

Figure 11. Chute of a granular mass along inclined planes. One inclined plane. Effects of the penalization parameters at two subsequent time instants. Arrows denote nodal velocities. Vectors length is proportional to their magnitude. Dark gray arrows are for $\epsilon=10^{5}$, light gray arrows for $\epsilon=10^{7}$.

The same parameters and mesh are then used to simulate the second test (two inclined planes, Figure $5 b$ ). A sequence of synchronized snapshots of the experimental and numerical runouts is comparatively shown in Figure 12. A view of the boundary of the final deposit in the $x-y$ plane at $t=2.5 \mathrm{~s}$ with $h_{\text {slip }}=0.04 \mathrm{~m}$ is also shown in Figure 13. Also in this case, a good agreement with the experiments can be observed.

\subsection{Chute of a granular mass on an erodible substrate}

In this example, the tests performed in [58] are simulated numerically. The chute of a mass of granular material along two planes of different inclination $\left(40^{\circ}\right.$ and $60^{\circ}$ respectively) is considered. A horizontal box is placed at the bottom of the inclined plane. Two different situations are investigated. In the first one, the material flows along the inclined plane and then it impacts against the empty box (see Figure 14(a)). In the second case, the box is filled with a small layer (5 $\mathrm{mm}$ ) of erodible granular material (see Figure 14(b)).

Also in this example the used material is a Hostun sand. Material parameters are provided in [58]: density $\rho=1420 \mathrm{Kg} / \mathrm{m}^{3}$, friction angle $\varphi=32^{\circ}$ and basal friction angle $\varphi_{\text {basal }}=25^{\circ}$. A viscosity of $\mu=1 \mathrm{~Pa} \mathrm{~s}$ is used also for these tests. The same material is used also for the erodible substrate. For the case without an erodible substrate, the following initial meshes have been used, with an average mesh size $h^{e}=0.007 \mathrm{~m}: 133318$ tetrahedra with 49477 nodes for the $40^{\circ}$ case; 133604 tetrahedra with 49529 nodes for the $60^{\circ}$ case. For the case with an erodible substrate, the following 


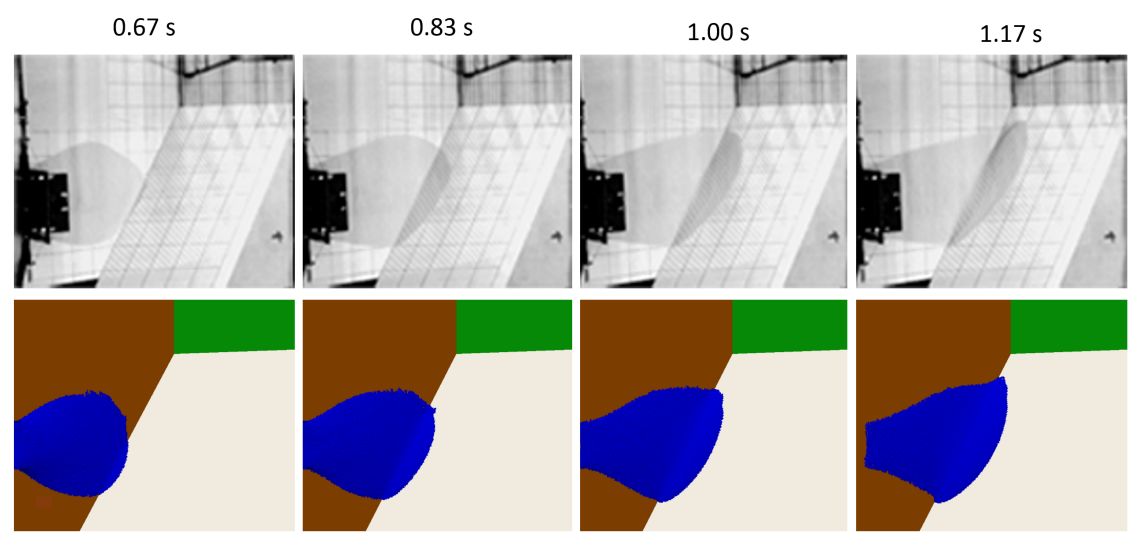

$1.33 \mathrm{~s}$

$1.50 \mathrm{~s}$

$1.67 \mathrm{~s}$

$1.83 \mathrm{~s}$
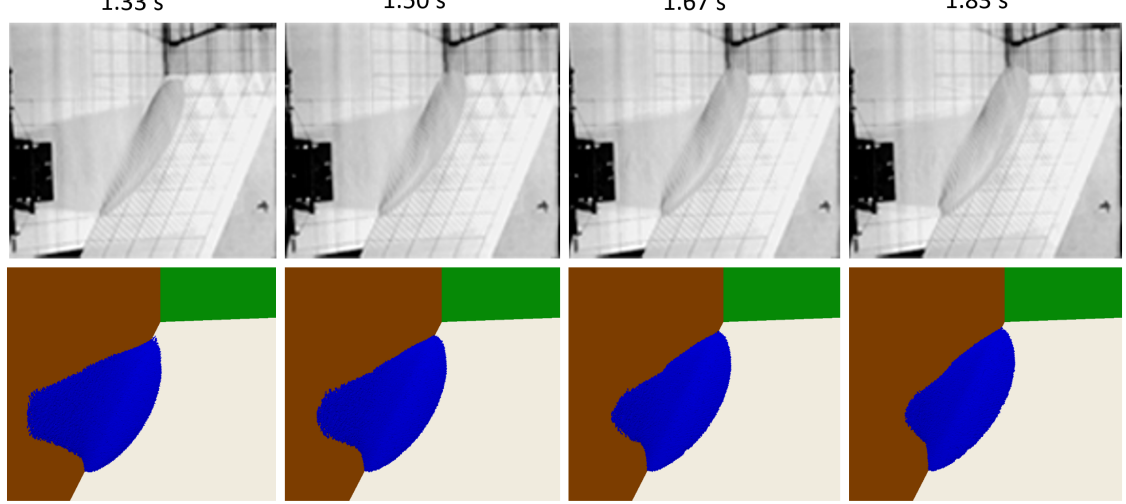

$2.00 \mathrm{~s}$
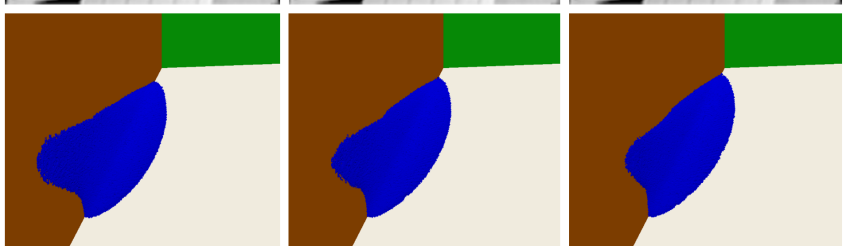

$2.17 \mathrm{~s}$

$2.33 \mathrm{~s}$

$2.50 \mathrm{~s}$
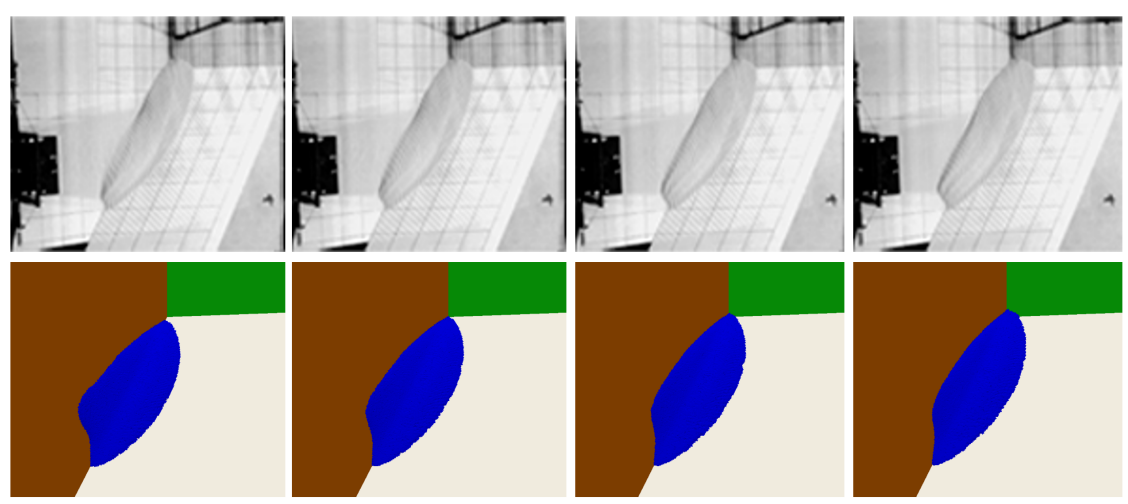

Figure 12. Chute of a granular mass along inclined planes. Two inclined planes. Comparison between experimental test (top) and numerical results (bottom) with $\mu=1 P a s$ and $h_{\text {slip }}=0.04 \mathrm{~m}$, at synchronized time instants.

initial meshes have been used, still with an average mesh size $h^{e}=0.007 \mathrm{~m}$ (the larger number of elements is due to the fact that also the erodible substrate need be discretized): 379243 tetrahedra with 94031 nodes for the $40^{\circ}$ case; 379548 tetrahedra with 95749 nodes for the $60^{\circ}$ case. A zero initial velocity has been prescribed for all nodes in the mesh.

In a first instance, the test with no erodible substrate has been performed with $h_{\text {slip }}=0$ (no slip at the interface). As shown in Figure 15, in the case with an inclination angle of $40^{\circ}$ after less than $0.2 \mathrm{~s}$, the front stops advancing, before the material has completely reached the horizontal plane, while in the case with $60^{\circ}$ the final front position differs significantly from the experimental data. 


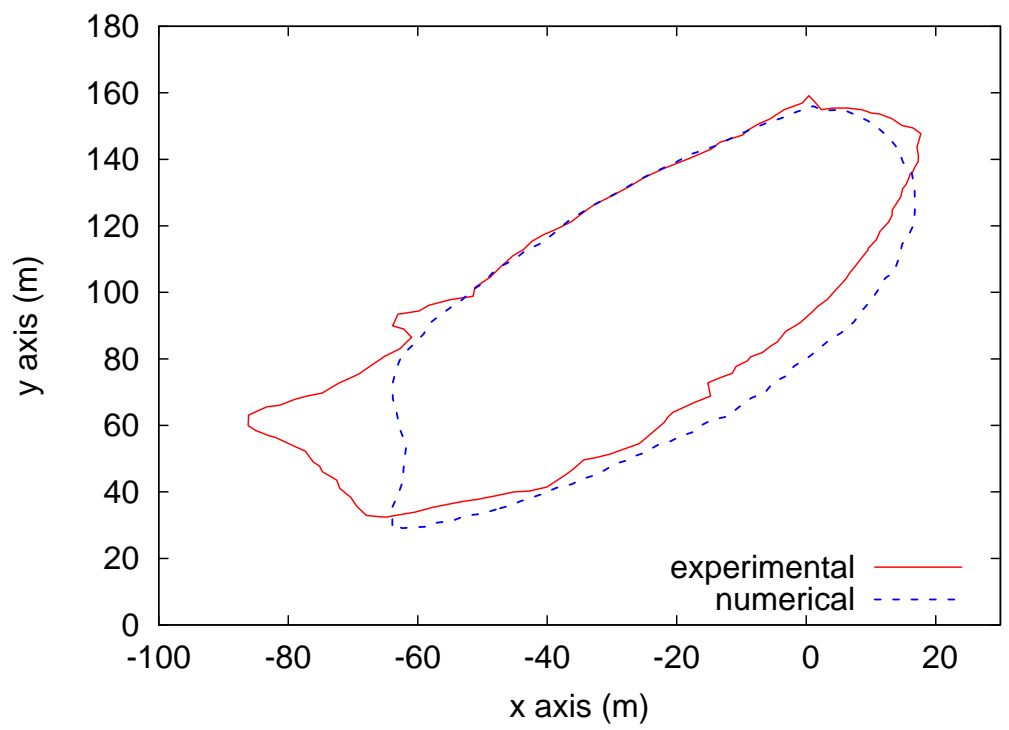

Figure 13. Chute of a granular mass along inclined planes. Two inclined planes. Comparison of the final deposits at $t=2.5 \mathrm{~s}$, with $\mu=1 \mathrm{~Pa}$ and $h_{\text {slip }}=0.04 \mathrm{~m}$.

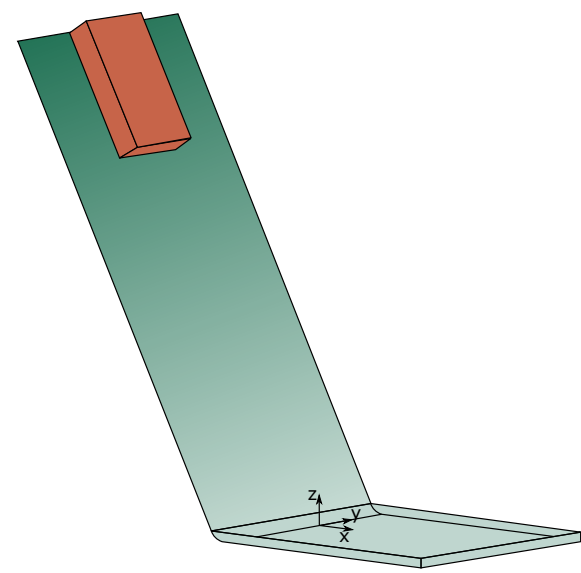

(a)

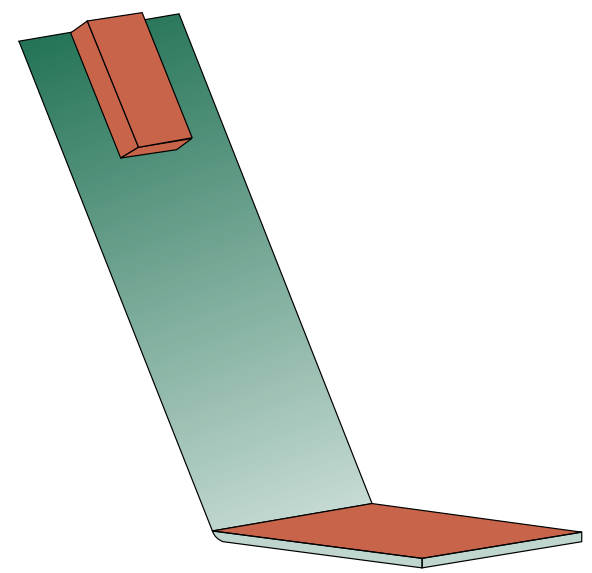

(b)

Figure 14. Chute of a granular mass on an erodible substrate. Initial position of sand mass: (a) without erodible substrate; (b) with erodible substrate.

Considering now the possibility of slip at the interface, the slip length should be identified. As in the previous example, the first test is used to calibrate the value $h_{\text {slip }}$, while the second is used to validate the results. The plot of Figure 16 shows the time evolution of the front distance for the first case (no erodible substrate), comparing numerical results and experimental data for the two plane inclinations. Two values of $h_{\text {slip }}$ are considered, showing that better results can be found using $h_{\text {slip }}=0.035 \mathrm{~m}$, with a ratio $h_{\text {slip }} / h^{e}=5$.

The front distance evolution for the second test (with erodible substrate), simulated using the parameters identified in the first test, is plotted in Figure 17. A satisfactory agreement is obtained also in this case for the $40^{\circ}$ inclination. In the $60^{\circ}$ case, the numerical solution seems to underestimate the run front distance. However, it should be noted that, in the case of the erodible substrate, the experimental identification of the front position is awkward due to the difficulty to distinguish between the granular material of the moving landslide and the one of the erodible substrate. Figure 18 shows snapshots with contour plots of the velocity magnitude at different time 


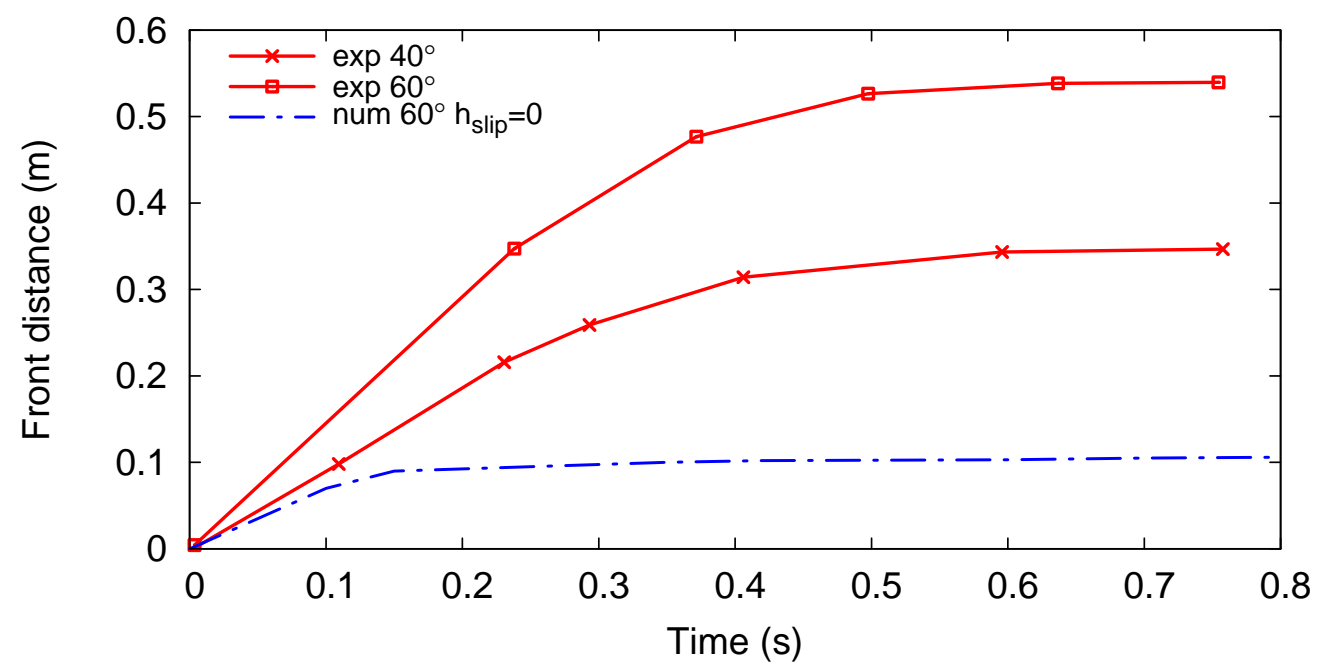

Figure 15. Chute of a granular mass on an erodible substrate. Front distance evolution. Results without slip at the interface.

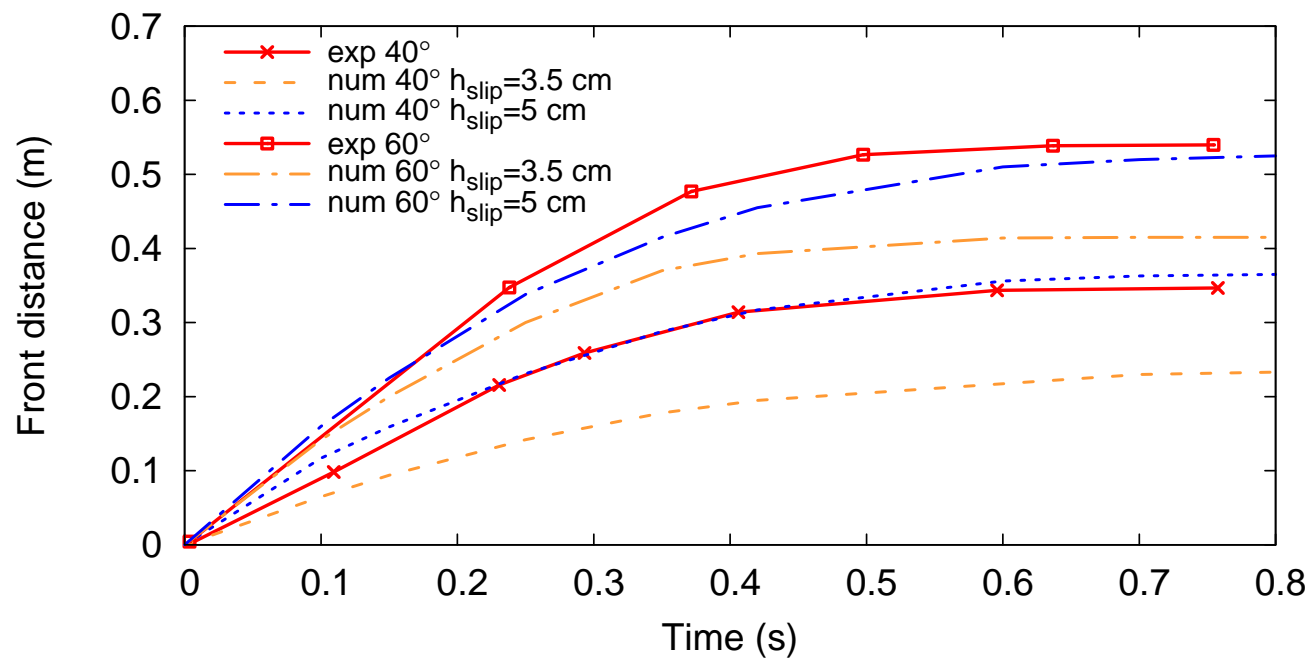

Figure 16. Chute of a granular mass on an erodible substrate. Front distance evolution. Test 1 (without erodible material).

instants, plotted on the vertical middle plane of the experimental setup. The effect of the slipping interface can be appreciated in the enlarged inserts, showing a non-zero relative velocity between the flowing mass and the basal plane. 


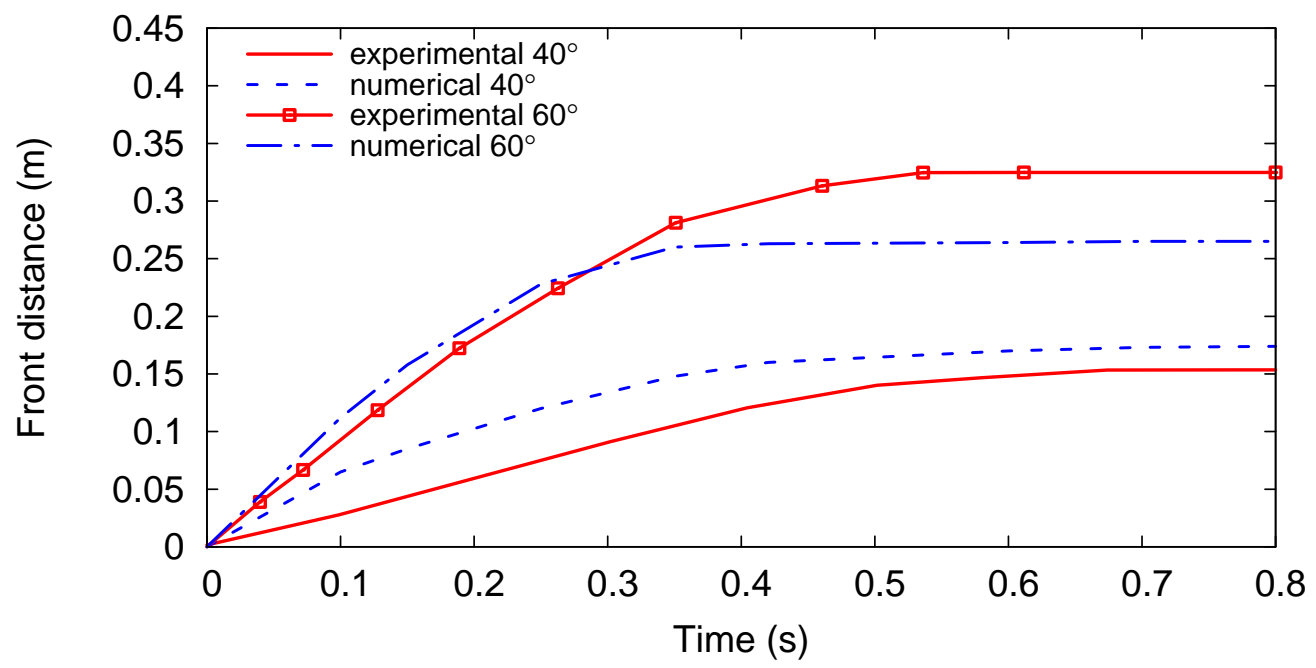

Figure 17. Chute of a granular mass on an erodible substrate. Front distance evolution. Test 2 (with erodible material, $h_{\text {slip }}=0.035 \mathrm{~m}$ ).
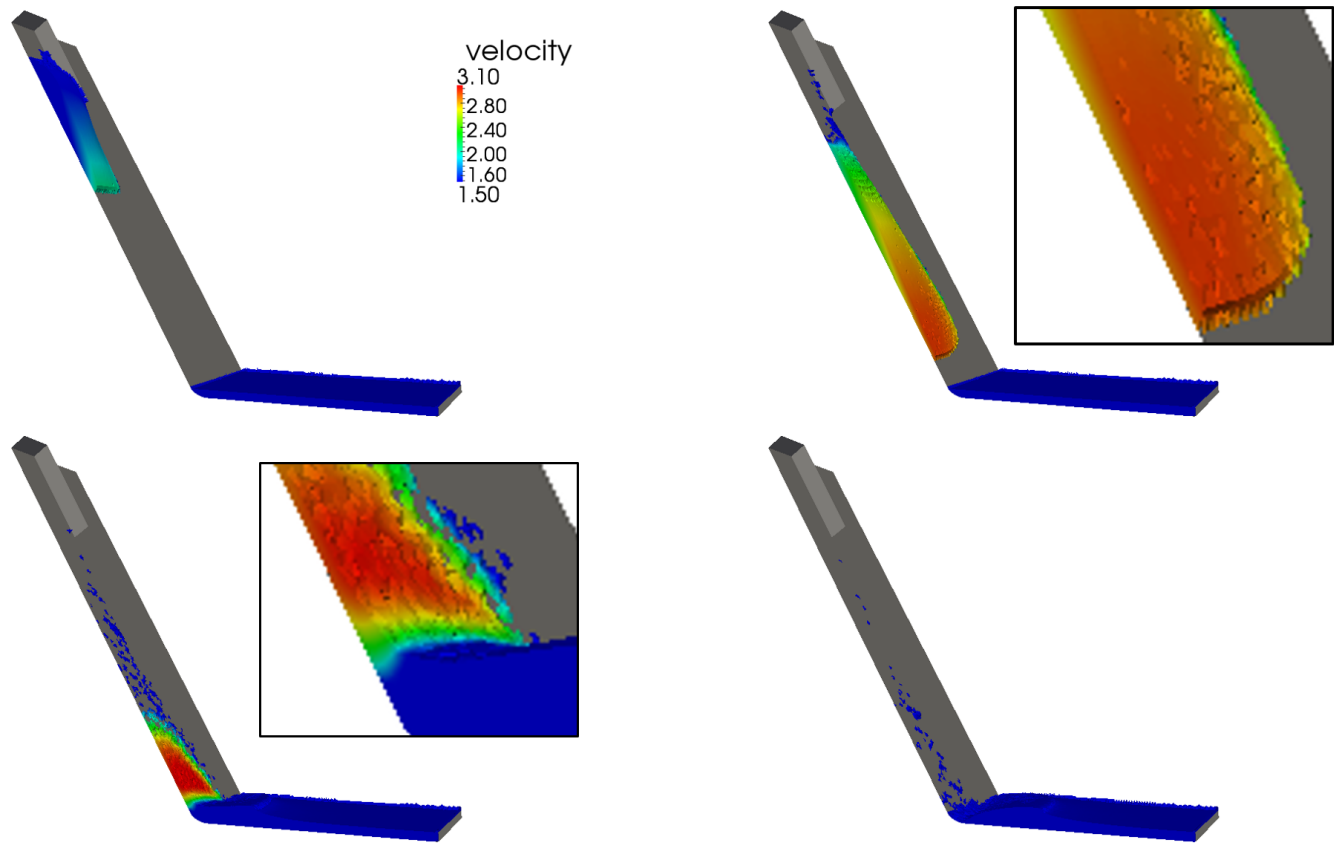

Figure 18. Chute of a granular mass on an erodible substrate. Test 2 (with erodible material). Snapshots at time instants $t=0.2 \mathrm{~s}, 0.4 \mathrm{~s}, 0.6 \mathrm{~s}$ and $0.8 \mathrm{~s}$ (from left to right, from top to bottom, respectively), with $h_{\text {slip }}=0.035 \mathrm{~m}$.

\subsection{Frank rock avalanche}

Frank slide (Canada, 1903) is considered as one of the most severe tragedies in Canada with a loss of approximately 70 lives. It involved more than 30 million of cubic meters of limestone rock detached from the ridge of Turtle Mountain, in the Northwest Territories. The landslide, with a $700 \mathrm{~m}$ wide front, descended along a $800 \mathrm{~m}$ slope creating a deposit $1.7 \mathrm{~km}$ wide and almost $2 \mathrm{~km}$ long. The deposit was approximately $18 \mathrm{~m}$ thick and the estimated duration of the event was of less than 100 
s. A detailed description of the event can be found in [59, 60]. A view of the final deposit is shown in Figure 19. The track followed by the running landslide is also clearly visible in the picture.

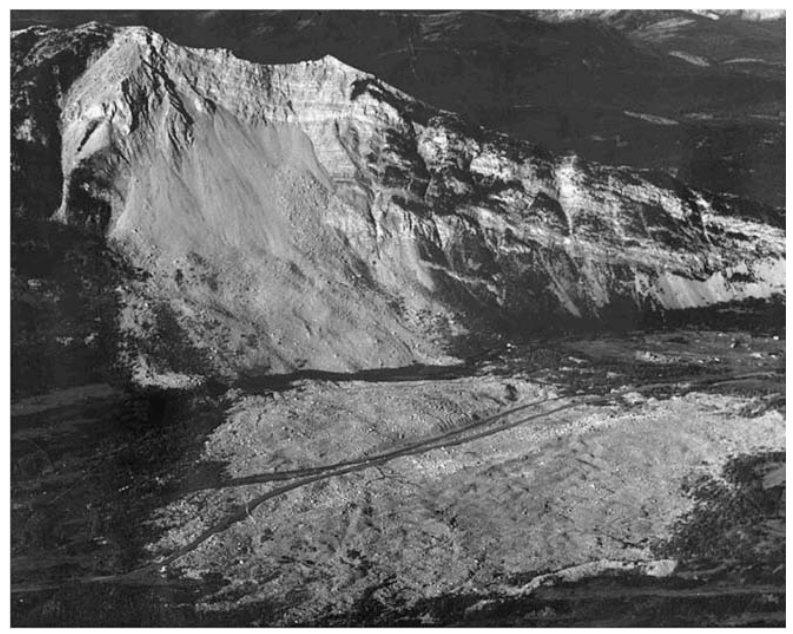

Figure 19. Frank rock avalanche. View of the final deposit [60].

Material properties are as in [12]: density $\rho=1800 \mathrm{Kg} / \mathrm{m}^{3}$, internal friction angle $\varphi=12.4^{\circ}$, viscosity $\mu=0.01 \mathrm{~Pa}$ s. A basal friction angle $\varphi_{\text {basal }}=\varphi=12.4^{\circ}$ has also been assumed. Four different values of the slip height have been considered: $h_{\text {slip }}=0 \mathrm{~m}, 20 \mathrm{~m}, 50 \mathrm{~m}$. The used initial mesh consists of 194795 tetrahedra, with 191028 nodes and an average mesh size $h^{e}=10 \mathrm{~m}$. A zero initial velocity has been prescribed for all nodes in the mesh.

Snapshots of the landslide runout are shown in Figure 20 at time instants $t=0 \mathrm{~s}, 14 \mathrm{~s}, 31 \mathrm{~s}, 71$ $\mathrm{s}$. The last snapshot shows the configuration of the final deposit and the corresponding time of $71 \mathrm{~s}$ compares well with the duration of less than $100 \mathrm{~s}$ reported by witnesses and with the propagation time of 80 s estimated in [12]. The profile of the final deposit is shown in Figure 21 for the different values of $h_{\text {slip }}$. Also in this case, the no-slip condition $h_{\text {slip }}=0$ leads to an underestimation of the runout distance, while better accuracy is achieved with the largest value $h_{\text {slip }}=50 \mathrm{~m}$, with a ratio $h_{\text {slip }} / h^{e}=5$. This large value of $h_{\text {slip }}$ can be explained with the very large (compared to the previous examples) average mesh size used in this case $\left(h^{e}=10 \mathrm{~m}\right)$. In fact, the slip condition directly affects an amount of flowing material which is of the order of magnitude of the mass in the elements in contact with the basal interface. This is confirmed by the observation that comparable values of the ratio $h_{s l i p} / h^{e}$ have provided excellent results in all the considered tests. Even though further studies are required, the present results seem to confirm that the slip length $h_{\text {slip }}$ to be used in the simulations is not only a property of the material/basal surface interface, but it should be also dependent on the non-dimensional factor $h_{s l i p} / h^{e}$, where $h^{e}$ is the typical size of a soil element on the basal interface. Typical values of this ratio that have provided good results in these tests are in the range $h_{\text {slip }} / h^{e}=4-5$.

\subsection{Conclusions}

In Lagrangian simulations of fast propagating landslides, the sliding soil mass is usually treated as a viscoplastic, Bingham-like, incompressible fluid, whose motion is governed by Navier-Stokes equations. For this type of fluid problems, the standard boundary conditions enforce zero relative slip between the fluid and the containing wall. These conditions are physically motivated for fluids, but have been shown to loose validity when the flowing material has a granular structure, in particular for increasing grain size.

In the present work, the soil material has been modeled as a rigid-viscoplastic, non-dilatant, Drucker-Prager material and specific slip conditions have been defined to model the interaction between the flowing mass and the basal surface. The new slip conditions are of Navier type, i.e. the resisting tangential force is proportional to the slip velocity. A pressure sensitive threshold has also 

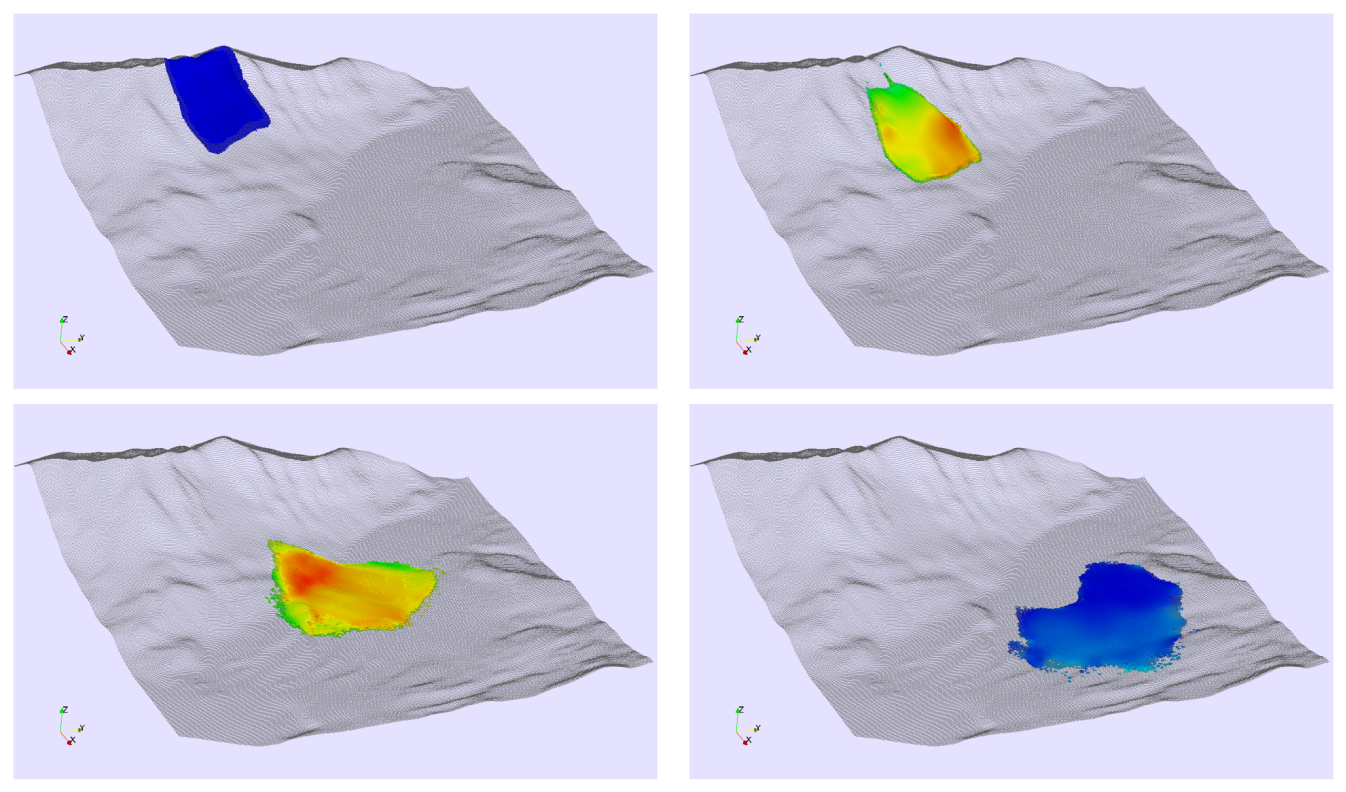

Figure 20. Frank rock avalanche. Snapshots at time instants $t=0 \mathrm{~s}, 14 \mathrm{~s}, 31 \mathrm{~s}, 71 \mathrm{~s}$ (from left to right, from top to bottom, respectively), with $h_{\text {slip }}=50 \mathrm{~m}$.

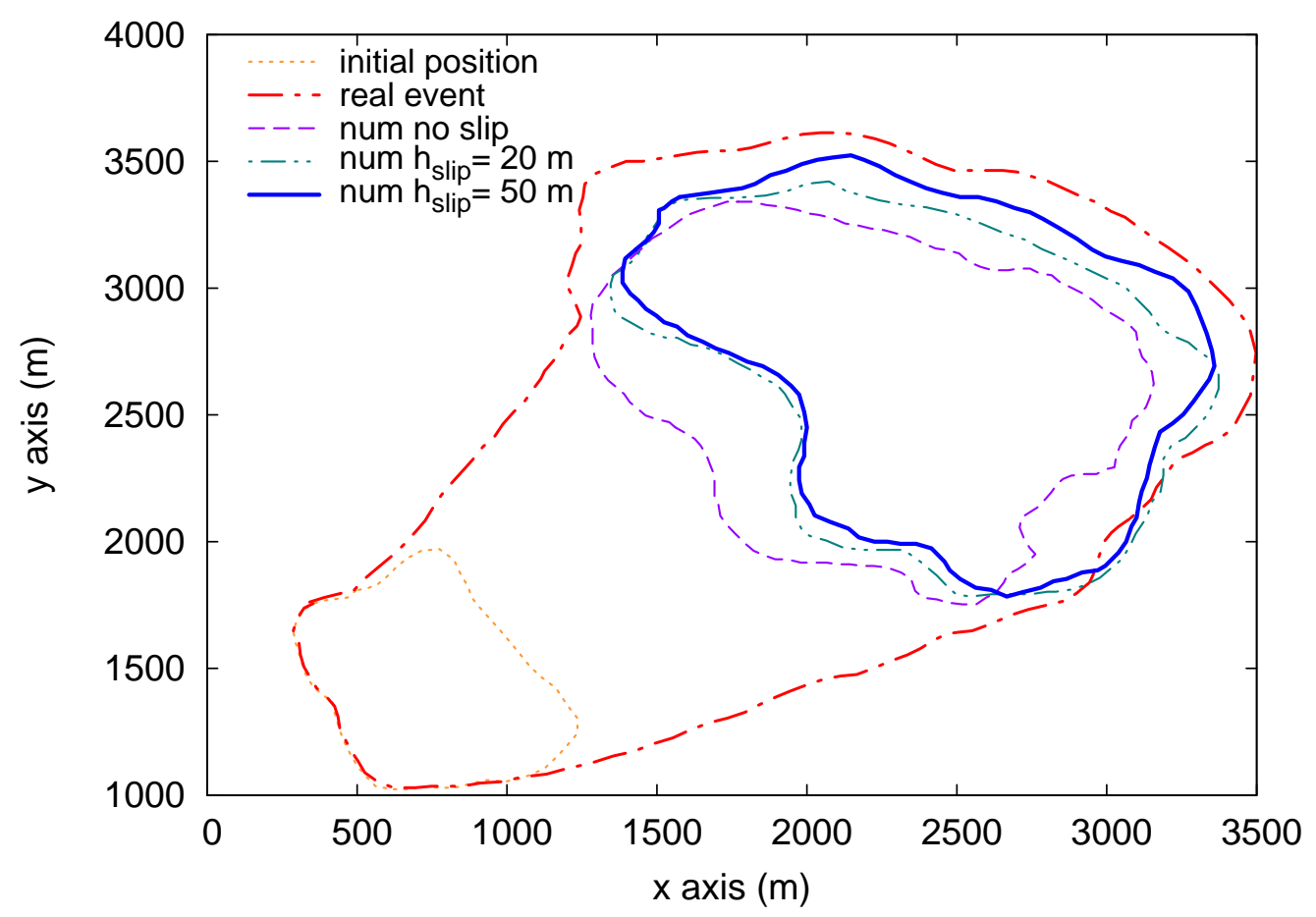

Figure 21. Frank rock avalanche. Landslide track and final deposit at $t=71 \mathrm{~s}$. Comparison between experimental observation and simulation with different slip lengths: $h_{s l i p}=0 \mathrm{~m}, 5 \mathrm{~m}, 20 \mathrm{~m}, 50 \mathrm{~m}$.

been introduced, so that the slip can occur only when the tangential stress exceeds the current limit value. The proposed interface law is characterized by two parameters: the slip height $h_{\text {slip }}$ and the basal friction angle $\varphi_{\text {basal }}$. 
The new boundary conditions require a special treatment to fit into the PFEM framework in view of the special role of the nodes on the basal surface, which are used to define the geometry of the computational domain and therefore cannot move. Finite elements having at least a node onto the basal surface are defined to have a mixed Lagrangian-Eulerian nature: nodes on the boundary are Eulerian, i.e. their velocity is set to zero and a convective term is added to the balance equation; interior nodes are Lagrangian, i.e. they move with the material velocity and the corresponding convective term is zero.

Slip boundary conditions need be supplemented with no-flux conditions, stating that the soil material cannot penetrate through the basal surface. These no-flux conditions are known to lead to inconsistencies when the surface is not smooth, due to ambiguity in the definition of the normal. The problem has been circumvented here by relaxing the no-flux condition by a penalty approach.

The importance of the slip conditions has been assessed by simulating four laboratory tests. In all cases, a proper definition of the slip parameter has allowed for a significant accuracy gain in the prediction of the runout distance and of the shape of the final deposit. The proposed numerical model has finally been applied to the simulation of a real large-scale landslide obtaining excellent accuracy in the prediction of the final deposit. In all the considered examples, best results have been obtained with values of the slip length $h_{\text {slip }}$ such that the ratio between the slip length and the average element size $h^{e}$ is in the range $h_{\text {slip }} / h^{e}=4-5$. However, these have to be considered only as preliminary results, while rigorous criteria for the identification of the $h_{\text {slip }}$ parameter have still to be investigated.

\subsection{Acknowledgments}

The Authors are grateful to prof. Giovanni Crosta and his group at Bicocca University, Milan, for providing data concerning their experiments on the chute of a granular mass on an erodible substrate. The Authors are also grateful to prof. Manuel Pastor and his group at the Universidad Politecnica de Madrid for providing the geometric data of Frank landslide.

\section{REFERENCES}

1. Calvetti F, Crosta G, Tartarella M. Numerical simulation of dry granular flows: from rhe reproduction of small-scale experiments to the prediction of rock avalanches. Rivista Geotecnica Italiana 2000; 34(2):21-38.

2. Taboada A, Estrada N. Rock-and-soil avalanches: Theory and simulation. Journal of Geophysical Research: Earth Surface 2009; 114(F3):1-23, doi:10.1029/2008JF001072.

3. Lu CY, Tang CL, Chan YC, Hu JC, Chi CC. Forecasting landslide hazard by the $3 \mathrm{~d}$ discrete element method: A case study of the unstable slope in the lushan hot spring district, central taiwan. Engineering Geology 2014; 183(0):1430, doi:10.1016/j.enggeo.2014.09.007.

4. Martelloni G, Bagnoli F, Massaro E. A computational toy model for shallow landslides: Molecular dynamics approach. Communications in Nonlinear Science and Numerical Simulation 2013; 18(9):2479 - 2492, doi: dx.doi.org/10.1016/j.cnsns.2012.11.026.

5. Pudasaini SP. A general two-phase debris flow model. Journal of Geophysical Research: Earth Surface 2012; 117(F3):1-28, doi:10.1029/2011JF002186.

6. Pitman EB, Le L. A two-fluid model for avalanche and debris flows. Philosophical Transactions of the Royal Society of London A: Mathematical, Physical and Engineering Sciences 2005; 363(1832):1573-1601, doi:10.1098/ rsta.2005.1596.

7. Savage SB, Hutter K. The motion of a finite mass of granular material down a rough incline. Journal of Fluid Mechanics 1989; 199(-1):177-215, doi:10.1017/s0022112089000340.

8. Hutter K, Koch T. Motion of a granular avalanche in an exponentially curved chute: Experiments and theoretical predictions. Philosophical Transactions of the Royal Society of London A: Mathematical, Physical and Engineering Sciences 1991; 334(1633):93-138, doi:10.1098/rsta.1991.0004.

9. Gray J, Wieland M, Hutter K. Gravity-driven free surface flow of granular avalanches over complex basal topography. Proceedings of the Royal Society A - Mathematical Physical \& Engineering Sciences 1999; 455:18411874, doi:10.1098/rspa.1999.0383.

10. Quecedo M, Pastor M, Herreros MI, Fernndez Merodo JA. Numerical modelling of the propagation of fast landslides using the finite element method. International Journal for Numerical Methods in Engineering 2004; 59(6):755-794, doi:10.1002/nme.841.

11. McDougall S, Hungr O. A model for the analysis of rapid landslide motion across three-dimensional terrain. Canadian Geotechnical Journal 2004; 41(6):1084-1097, doi:10.1139/t04-052.

12. Pastor M, Blanc T, Haddad B, Drempetic V, Morles MS, Dutto P, Stickle MM, Mira P, Merodo JaF. Depth Averaged Models for Fast Landslide Propagation: Mathematical, Rheological and Numerical Aspects. Archives of Computational Methods in Engineering 2015; 22:67-104, doi:10.1007/s11831-014-9110-3. 
13. Pastor M, Haddad, Sorbino B, Cuomo S, Drempetic V. A depth-integrated, coupled SPH model for flow-like landslides and related phenomena. International Journal for Numerical and Analytical Methods in Geomechanics 2009; 33:143-172, doi:10.1002/nag.705.

14. Berger M, George D, LeVeque R, Mandli K. The geoclaw software for depth-averaged flows with adaptive refinement. Advances in Water Resources 2011; 34(9):1195-1206, doi:10.1016/j.advwatres.2011.02.016.

15. Pitman EB, Nichita CC, Patra A, Bauer A, Sheridan M, Bursik M. Computing granular avalanches and landslides. Physics of Fluids 2003; 15(12):3638-3646, doi:10.1063/1.1614253.

16. Beguería S, Van Asch TWJ, Malet JP, Gröndahl S. A gis-based numerical model for simulating the kinematics of mud and debris flows over complex terrain. Natural Hazards and Earth System Science 2009; 9(6):1897-1909, doi:10.5194/nhess-9-1897-2009.

17. Wang F, Sassa K. Landslide simulation by a geotechnical model combined with a model for apparent friction change. Physics and Chemistry of the Earth 2010; 35(3-5):149-161, doi:10.1016/j.pce.2009.07.006.

18. Chen H, Lee C. A dynamic model for rainfall-induced landslides on natural slopes. Geomorphology 2003; 51(4):269-288, doi:10.1016/S0169-555X(02)00224-6.

19. Crosta G, Imposimato S, Roddeman D. Numerical modelling of entrainment/deposition in rock and debrisavalanches. Engineering Geology 2009; 109(12):135 - 145, doi:10.1016/j.enggeo.2008.10.004.

20. Andersen S, Andersen L. Modelling of landslides with the material-point method. Computational Geosciences 2010; 14(1):137-147, doi:10.1007/s10596-009-9137-y.

21. Hungr O. A model for the runout analysis of rapid flow slides, debris flows, and avalanches. Can. Geotech. J. 1995; 32(4):610-623, doi:10.1139/t95-063.

22. McDougall S, Pirulli M, Hungr O, Scavia C. Advances in landslide continuum dynamic modelling. CRC Press, 2008; 145-157, doi:10.1201/9780203885284-c8.

23. Lagrée PY, Staron L, Popinet S. The granular column collapse as a continuum: validity of a two-dimensional NavierStokes model with a (I)-rheology. Journal of Fluid Mechanics 2011; 686:378-408, doi:10.1017/jfm.2011. 335.

24. Pastor M, Blanc T, Pastor MJ. A depth-integrated viscoplastic model for dilatant saturated cohesive-frictional fluidized mixtures: Application to fast catastrophic landslides. Journal of Non-Newtonian Fluid Mechanics 2009; 158:142-153, doi:10.1016/j.jnnfm.2008.07.014.

25. Crosta G, Imposimato S, Roddeman D. Numerical modelling of entrainment/deposition in rock and debrisavalanches. Engineering Geology 2009; 109(12):135 - 145, doi:dx.doi.org/10.1016/j.enggeo.2008.10.004.

26. Cremonesi M, Frangi A, Perego U. A lagrangian finite element approach for the simulation of water-waves induced by landslides. Computers and Structures 2011; 89(5):1086-1093, doi:10.1002/nme.2911.

27. Oñate E, Idelsohn S, Del Pin F, Aubry R. The particle finite element method. An overview. International Journal of Computational Methods 2004; 1(2):267-307, doi:10.1142/S0219876204000204.

28. Idelsohn S, Oñate E, Del Pin F. The particle finite element method: a powerful tool to solve incompressible flows with free-surfaces and breaking waves. International Journal for Numerical Methods in Engineering 2004; 61(7):964-989, doi:10.1002/nme.1096.

29. Idelsohn SR, Oñate E, Pin FD, Calvo N. Fluidstructure interaction using the particle finite element method. Computer Methods in Applied Mechanics and Engineering 2006; 195(17-18):2100-2123, doi:10.1016/j.cma.2005. 02.026.

30. Idelsohn SR, Marti J, Limache A, Oñate E. Unified lagrangian formulation for elastic solids and incompressible fluids: Application to fluidstructure interaction problems via the PFEM. Computer Methods in Applied Mechanics and Engineering 2008; 197(19-20):1762-1776, doi:10.1016/j.cma.2007.06.004.

31. Cremonesi M, Frangi A, Perego U. A lagrangian finite element approach for the analysis of fuid-structure interaction problems. International Journal for Numerical Methods in Engineering 2010; 84(5):610-630, doi: $10.1002 / n m e .2911$.

32. Zhang X, Krabbenhoft K, Sheng D, Li W. Numerical simulation of a flow-like landslide using the particle finite element method. Computational Mechanics 2015; 55(1):167-177, doi:10.1007/s00466-014-1088-z.

33. Salazar F, Irazábal J, Larese A, Oñate E. Numerical modelling of landslide-generated waves with the particle finite element method (PFEM) and a non-Newtonian flow model. International Journal for Numerical and Analytical Methods in Geomechanics 2015; doi:10.1002/nag.2428.

34. Denn MM. Extrusion instabilities and wall slip. Annual Review of Fluid Mechanics 2001; 33(1):265-287, doi: 10.1146/annurev.fluid.33.1.265.

35. Schowalter WR. The behavior of complex fluids at solid boundaries. Journal of Non-Newtonian Fluid Mechanics Jan 1988; 29:25-36, doi:10.1016/0377-0257(88)85048-1.

36. Schaefer M, Bugnion L, Kern M, Bartelt P. Position dependent velocity profiles in granular avalanches. Granular Matter 2010; 12:327-336, doi:10.1007/s10035-010-0179-6.

37. Lucas A, Mangeney A, Ampuero JP. Frictional velocity-weakening in landslides on earth and on other planetary bodies. Nature Communications 2014; 5, doi:10.1038/ncomms4417.

38. Habib P. Production of gaseous pore pressure during rock slides. Rock mechanics 1975; 7(4):193-197, doi: $10.1007 / \mathrm{bf} 01246865$

39. Voight B, Faust C. Frictional heat and strength loss in some rapid landslides. Géotechnique 1982; 32(1):43-54, doi:10.1680/geot.1982.32.1.43.

40. Vardoulakis I. Dynamic thermo-poro-mechanical analysis of catastrophic landslides. Géotechnique 2002; 52(3):157-171, doi:10.1680/geot.2002.52.3.157.

41. Cecinato F, Zervos A, Veveakis E. A thermo-mechanical model for the catastrophic collapse of large landslides. Int. J. Numer. Anal. Meth. Geomech. 2011; 35(14):1507-1535, doi:10.1002/nag.963.

42. Richardson S. On the no-slip boundary condition. Journal of Fluid Mechanics 1973; 59(04):707-719, doi: $10.1017 / \mathrm{s} 0022112073001801$.

43. Thompson PA, Troian SM. A general boundary condition for liquid flow at solid surfaces. Nature 1997; 389(6649):360-362, doi:10.1038/38686. 
44. Thompson PA, Robbins MO. Shear flow near solids: Epitaxial order and flow boundary conditions. Physical Review A 1990; 41(12):6830-6837, doi:10.1103/physreva.41.6830.

45. Fortin A, Coté D, Tanguy PA. On the imposition of friction boundary conditions for the numerical simulation of bingham fluid flows. Computer Methods in Applied Mechanics and Engineering 1991; 88:97-109.

46. Papanastasiou TC. Flows of materials with yield. Journal of Rheology 1987; 31(5):385-404, doi:10.1122/1.549926.

47. Matthews MT, Hill JM. Newtonian flow with nonlinear navier boundary condition. Acta Mechanica 2007; 191(34):195-217, doi:10.1007/s00707-007-0454-8.

48. Verfürth R. Finite element approximation of incompressible Navier-Stokes equations with slip boundary condition II. Numerische Mathematik 1991; 59(1):615-636, doi:10.1007/bf01385799.

49. Engelman MS, Sani RL, Gresho PM. The implementation of normal and/or tangential boundary conditions in finite element codes for incompressible fluid flow. Int. J. Numer. Meth. Fluids 1982; 2(3):225-238, doi: 10.1002/fld.1650020302.

50. Behr M. On the application of slip boundary condition on curved boundaries. Int. J. Numer. Meth. Fluids 2004; 45(1):43-51, doi:10.1002/fld.663.

51. Dione I, Tibirna C, Urquiza J. Stokes equations with penalised slip boundary conditions. International Journal of Computational Fluid Dynamics 2013; 27(6-7):283-296, doi:10.1080/10618562.2013.821114.

52. Donea J, Huerta A. Finite Element Methods for Flow Problems. Wiley, 2003.

53. Simo J, Hughes T. Computational Inelasticity. Springer, 2000.

54. Brezzi F, Fortin M. Mixed and Hybrid Finite Element Method. Springer-Verlag, 1991

55. Tezduyar TE, Mittal S, Ray SE, Shih R. Incompressible flow computations with stabilized bilinear and linear equalorder-interpolation velocity-pressure elements. Computer Methods in Applied Mechanics and Engineering 1992; 95(2):221-242, doi:10.1016/0045-7825(92)90141-6.

56. Ferrás LL, Nóbrega JM, Pinho FT. Analytical solutions for Newtonian and inelastic non-Newtonian flows with wall slip. Journal of Non-Newtonian Fluid Mechanics 2012; 175-176:76-88, doi:10.1016/j.jnnfm.2012.03.004.

57. Manzella I. Dry rock avalanche propagation: unconstrained flow experiments with granular materials and small bricks. PhD Thesis, Ecole Polytechnique Fédéral de Lausanne 2008.

58. Crosta G, De Blasio F, De Caro M, Volpi G, Imposimato S, Roddeman. Model of collapse and propagation of a granular mass onto an erodible substrate. submitted for pubblication 2014;

59. Cruden DM, Hungr O. The debris of the frank slide and theories of rockslideavalanche mobility. Canadian Journal of Earth Sciences 1986; 23(3):425-432, doi:10.1139/e86-044.

60. Wang F, Sassa K. Landslide simulation by a geotechnical model combined with a model for apparent friction change. Physics and Chemistry of the Earth, Parts A/B/C 2010; 35(3-5):149-161, doi:10.1016/j.pce.2009.07.006. 